\title{
CONSTRUCTION PHASE ORIENTED DYNAMIC SIMULATION: TAKING RCC DAM PLACEMENT PROCESS AS AN EXAMPLE
}

\author{
Wei HU, Denghua ZHONG, Binping WU(1), Zheng LI \\ State Key Laboratory of Hydraulic Engineering Simulation and Safety, Tianjin University, Tianjin 300072, China \\ Received 28 September 2018; accepted 29 November 2018
}

\begin{abstract}
Construction simulation has been widely applied in schedule analysis. However, traditional simulation is based on static models built in the planning or design phase, which focuses on overall project-level schedule analysis. To provide activity-level simulation for on-site schedule management, a construction phase oriented dynamic simulation method is proposed, which takes roller compacted concrete (RCC) dam placement process as an example. Considering various innerlayer and inter-layer activities and different construction organization modes, a detailed placement process simulation model is built. Based on construction data collected by real-time monitoring, a construction activity modeling method is given. Additionally, Dirichlet process mixture (DPM) models are applied for simulation parameter updates, which endows density estimation with considerable flexibility and robustness. A fast inference algorithm is also proposed to realize the fast posterior computation of DPM models. The proposed method is tested by an RCC dam project in southwest China. The results show that the proposed method can reflect the dynamic features of the actual placement process in the construction phase and provide accurate schedule predictions for on-site construction management.
\end{abstract}

Keywords: construction phase, dynamic simulation, RCC dam, placement process, real-time monitoring, DPM models.

\section{Introduction}

RCC dams feature a high mechanization level, fast construction speed, and less hydration heat and have been widely applied in recent water conservancy projects. The overall RCC dam construction process can be divided into concrete production, transportation, and placement. Concrete placement is the most complex among the stages, which involves the organization of concentrated resources, equipment and labor among several construction activities. Because the construction speed of the placement process decides the overall schedule of the RCC dam project, it is critical to have an effective construction schedule prediction and analysis method for the placement process.

Simulation has been proven to be a powerful tool for construction schedule analysis, which can conveniently investigate different construction plans and provide useful information for management (Abdallah \& Marzouk, 2013; Gurgun, Zhang, \& Touran, 2013; Han, Ko, Hong, Koo, \& Lee, 2016). Since Halpin (1977) proposed the cycle operation network (CYCLONE) construction simulation method for repetitive projects, there have been various simulation methods including UM-CYCLONE (Ioannou, 1989), Micro-CYClONE (Halpin, 1990), MODSIM (Oloufa,
1993), STROBOSCOPE (Martinez \& Ioannou, 1994), Simphony (AbouRizk \& Mohamed, 2000), COSYE (AbouRizk \& Hague, 2009), hybrid SD-DES (Alvanchi, Lee, \& AbouRizk, 2011) and ABMS (Son, Rojas, \& Shin, 2015). For RCC dam construction, Wang, Zhong, and Zha (1995) investigated the RCC dam construction simulation using the system simulation and queue stochastic simulation network. Luo, Liu, and $\mathrm{Hu}$ (2009) built a petri net-based simulation model to study the coupling relationship between the production, transportation and placement processes of RCC dams. Zhao, Dong, and Zhou (2013) studied the concrete placement process system and construction scheme optimization under limited-resource conditions for RCC dams. Wang, Zhong, Wu, Yu, and Chang (2018) proposed an RCC dam construction simulation method that updates the simulation model based on real-time monitoring and Bayesian updating.

Although the above simulation methods promote RCC dam construction schedule analysis, studies on detailed simulation of the complex concrete placement process are rare. The placement strength is taken as a fixed empirical value or set equal to the transportation strength.

\footnotetext{
*Corresponding author. E-mail: binpingwu@hotmail.com
} 
Study by Zhao et al. (2013) simplified the placement process by considering only horizontal-layer construction activities, while there are also slopping-layer organizations forming and interlayer activities in the actual placement process. For on-site construction management, simulation tools that reflect the actual placement procedure are badly needed.

In addition, previous studies on RCC dam construction simulation are mainly planning or design phase oriented and are built on static simulation models. Static simulation models use parameters estimated according to similar projects or expert experience, which is not precise enough for short-term on-site schedule analysis. In addition, RCC dam placement is a dynamic process affected by environmental factors such as temperature, rainfall, and season. Even though the static simulation model can match the construction for some time, it will deviate from the actual process as time goes on. To overcome the deficiency of static simulations, data-driven dynamic construction simulation has been investigated by researchers with the development of construction data collection and construction process monitoring technology. Dynamic data-driven application simulation (DDDAS) was put forward by Darema (2004) for the first time, and it emphasized incorporating archival or collected on-line data to enhance and refine the original application model, and the application also has the ability to control and guide the processes for more effective measurements. Lu, Dai, and Chen (2007) used the machinery working time data acquired from a vehicle track system to update simulation inputs and established the HKCONSIM-Realtime system for concrete projects. Song and Eldin (2012) introduced an adaptive modeling method for heavy construction work simulation, which updated simulation parameters and simulation logics based on real-time monitoring data and background knowledge. Akhavian and Behzadan (2012) proposed a data-driven modeling framework that collects and manipulates real-time field data from construction equipment to create dynamic 3D visualizations of ongoing activities and to update a discrete event simulation model. These studies proved that applying the DDDAS framework to construction simulation by collecting actual construction data to update the simulation model is a feasible way to realize dynamic simulations. To bridge the gap between the dynamic simulation model and the captured construction data, detailed construction activity recognition and state identification were also investigated. Akhavian and Behzadan (2013) proposed a multimodal process datamining algorithm to extract operational knowledge of construction fleet operations and automatically update the discrete event simulation model. Vahdatikhaki and Hammad (2014) put forward a set of multidimensional rules accommodating various types of $2 \mathrm{D}$ and $3 \mathrm{D}$ sensory data for earthmoving equipment state identification in a near real-time simulation framework. Akhavian and Behzadan (2015) extracted key features from data collected by builtin mobile sensors and trained supervised machine learning classifiers to recognize construction activities, which could be further used for simulation model input parameter verification and updating. Concerning RCC dam dynamic construction simulations, Wang et al. (2018) research on real-time monitoring-based simulation is a kind of DDDAS. However, the research is about the overall dam process, and a dynamic construction simulation for the placement process is still badly needed for on-site schedule management.

Additionally, proper estimation of simulation parameters is a key problem in construction simulation, especially for near-future schedule prediction in the construction phase. Because a simulation is a computer-based statistical sampling experiment (Law, 2015), the sampling basis, i.e., the density of the simulation parameters, directly influences the simulation accuracy. To accurately capture actual construction features, studies have focused on effective simulation parameter estimations. Chung, Mohamed, and Abourizk (2006) applied Bayesian updating technology to update the distribution of simulation parameters, which improved the simulation accuracy. Zhang, Du, Sa, C. Wang, and G. Wang (2014) adopted the Bayesian method to constantly update the duration distributions of unfinished project activities and simulated the completion probability in underground cavern group projects. Guan, Zhong, Ren, Song, and Chu (2018) applied the fuzzy set theory to describe simulation parameters updated by the Bayesian method. The application of the Bayesian method provides a useful means for incorporating actual construction data. However, these methods all assume that construction parameters obey a normal distribution, which is reasonable according to the central limit theory for longterm construction. However, for the short-term on-site construction analysis, construction parameter distributions are complex and feature multiple peaks and timely variation, of which the scope and type cannot be accurately described by normal or other classical distributions.

In this paper, aiming at the above problems, a construction phase oriented simulation method for the RCC dam placement process is proposed. First, a detailed RCC dam placement process simulation model is built. The model not only considers organization forms choosing between the horizontal layer or slopping layer and the flow process or sequence among inner-layer activities but also takes interlayer activities such as mortar paving, the cooling pipes layout, and bedding mortar paving into consideration. Second, based on actual construction data collected by the real-time monitoring system (Liu, Zhong, Cui, Zhong, \& Wei, 2015), a detailed construction activity analysis and modeling method is proposed. Both inner-layer and inter-layer activities are modeled according to their characteristics and accessible data, especially presenting a meticulous discrete time model for rolling, which is the key activity of the placement process. Third, Dirichlet process mixture (DPM) models are applied to accurately estimate the simulation parameter density. DPM models, proposed by Antoniak (1974) and based on Ferguson's introduction of Dirichlet processes, are research hotspots in the field of nonparametric Bayesian methods (Lo, 1984; 
Escobar, 1994; Escobar \& West, 1995). DPM models do not constrain the inference to a specific parametric form but put the inference into an infinite-dimensional space that allows modeling flexibility and robustness, which is suitable for complex density estimations. However, this flexibility also brought considerable computation difficulty until Monte-Carlo Markov chain (MCMC) methods made posterior inference tractable. There are various MCMC-based algorithms, including marginal Gibbs sampling (MacEachern, 1994; West \& Escobar, 1993), conditional Gibbs sampling (Ishwaran \& James, 2001), and split-merge (Jain \& Neal, 2004). However, MCMC-based algorithms are still time-consuming. Processing a moderately sized dataset can even cost several hours, which is not suitable to be applied in a real-time streaming data situation. To realize simulation parameter quick estimation, an improved sequential updating and greedy search (SUGS) algorithm is proposed for fast inference in DPM models.

The paper is organized as follows: Section 1 gives the framework of construction phase oriented simulation for RCC dam placement process. Section 2 builds the detailed simulation model for RCC dam placement process. Section 3 proposes construction activity analysis and modeling method based on real-time monitoring data. Section 4 applies DPM models to simulation parameters' density estimation and proposes a fast online inference algorithm. In section 5, an application of a RCC dam project in west China is used to test the effectiveness of proposed simulation method.

\section{Framework of construction phase oriented dynamic simulation}

The framework of the construction phase oriented dynamic simulation for the RCC dam placement process is described in Figure 1.

First, a detailed placement process simulation model is built based on the actual construction process. RCC dam placement is a complex process involving several organization modes among multiple activities and resources. The construction phase-oriented dynamic simulation demands that the simulation model contains details about on-site construction. The proposed placement process simulation model considers different organizational forms among the construction activities. Horizontal- or slopping-layer construction is chosen to divide the placement unit into several constructible layers by comprehensively considering the permissible time intervals between placing layers and the concrete supply and placement ability. For layer construction, considering machinery sizes and placement unit widths, flow-process construction or sequence construction is adopted to maximize the construction speed. The total layer construction duration under the flow process or sequence construction is proposed. In addition, the final placement unit construction duration not only contains each layer's duration but also takes the interlayer activities' construction times into consideration.

However, a detailed simulation model alone is still a static model, which cannot realize dynamic schedule prediction for on-site management. To capture the dynamics

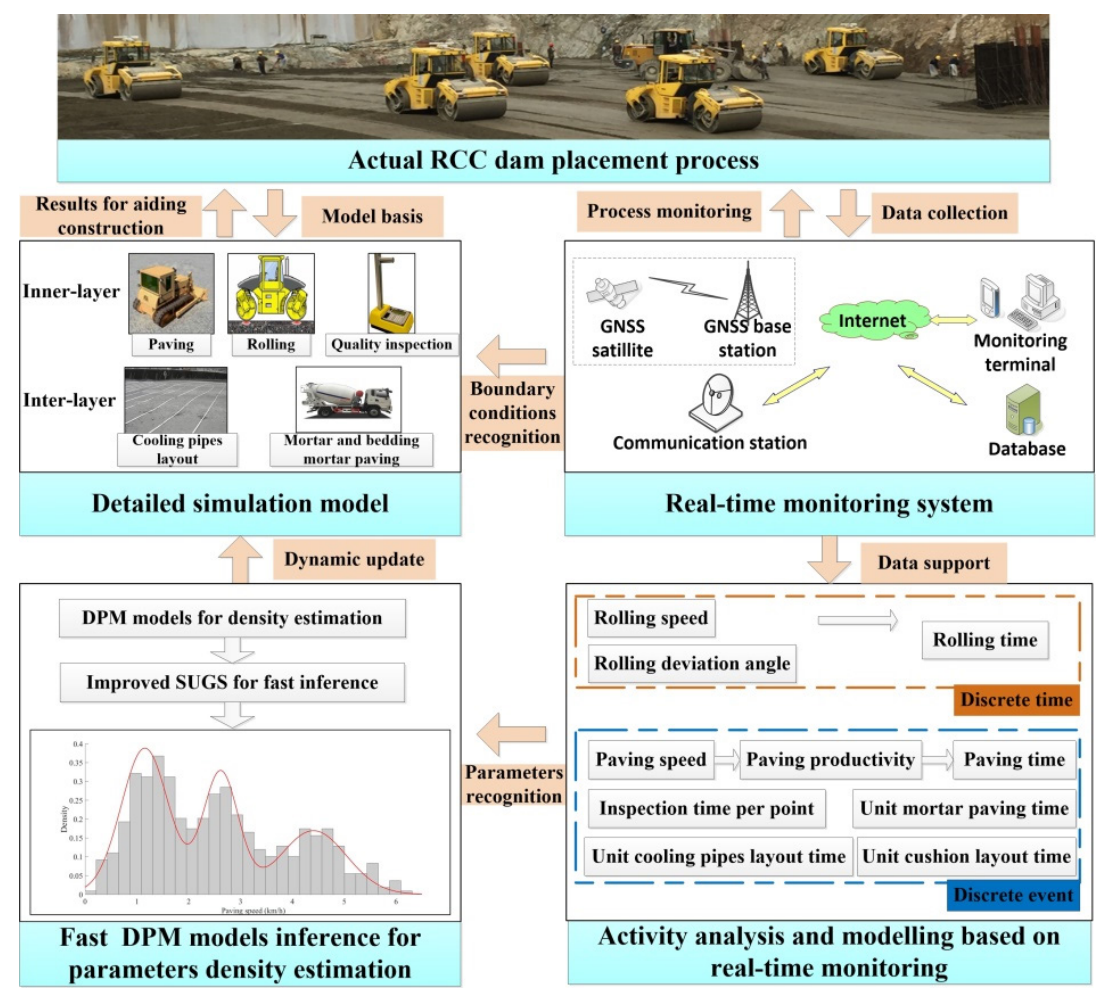

Figure 1. Framework of construction phase oriented dynamic simulation for RCC dam placement process 
of the construction process, an RCC dam storehouse surface real-time monitoring system based on the research by Liu et al. (2015) is applied. Placement unit real-time monitoring is designed to control construction process quality through real-time monitoring of working machinery. It brings an effective way for automatic construction data collection, which can be further analyzed to capture dynamic patterns of construction activities. Additionally, simulation boundary conditions such as placement unit sizes, resource supplies, and current construction states, can be directly acquired from the real-time monitoring system.

With the support of real-time data, a construction activity modeling method is also proposed. Based on collected rolling speeds and rolling deviation angles, rolling activities are modeled by the discrete time simulation method. As the simulation clock advances, the rolling passes of each grid in the layer are calculated. When the first rolling is completed and the rolling area standard rolling times ratio is not up to the standard, additional rolling is arranged, which brings an additional time cost to the rolling process. Other construction activities including paving, quality inspection, mortar paving, cooling pipes layout, and bedding mortar paving are also modeled by random distributions based on real-time monitoring data.

Construction activity modeling based on real-time monitoring points out that basic simulation parameters such as paving speed, rolling speed, and unit inspection time need to be accurately updated. Considering simulation parameters' complexity and dynamics in actual construction, DPM models are used to estimate simulation parameter densities on an infinite-dimensional space. In addition, an improved SUGS method is applied for fast inference of DPM models under real-time streaming data, which realizes the dynamic updating of simulation parameter densities.

The dynamically updated parameters together with boundary conditions can be requested by the placement process simulation model at any time. The simulation results are used to aid in on-site placement process schedule management, which, in turn, influences the actual placement unit construction process.

\section{Detailed simulation model establishment}

\subsection{Construction process analysis}

RCC dams consist of multiple dam blocks along the vertical direction of river flow. The placement unit, the basic construction unit, is composed of several adjacent dam blocks at a certain altitude interval (typically 3-6 m).

To meet the needs of high-speed construction, placement process construction typically adopts a horizontal layer or slopping layer organizational form. Horizontal layer construction divides the placement unit into several $30 \mathrm{~cm}$-thickness layers along the direction of the elevation. However, when the placement unit area is relatively large, the capacity of concrete production and pouring cannot support finishing the horizontal layer construction within the permissible time interval between placing layers. To avoid layer combination quality problems resulting from exceeding the time interval, slopping-layer construction, which sets inclined layers to reduce the construction area, is adopted in this situation.

A layer is a basic construction activity organizational unit. The main construction activities in a layer include paving, rolling, lancing, and quality inspection. When a placement unit's width is relatively large, flow-process construction is applied. The layer is divided into several construction sections, and paving, rolling and quality inspection are conducted for each section in turn. Otherwise, sequential construction is applied, in which construction activities are conducted for the whole layer in turn.

In addition to the above inner-layer construction activities, there are also interlayer processes. To guarantee the joint quality with previous final condensation concrete, it is necessary to pave the working surface with a thin layer of mortar before construction of the placement unit's first layer. After the placement of every five layers, cooling pipes should be laid out on the completed layer, preparing for water cooling inside the concrete. If the layer's construction time (including the cooling pipes layout time) exceeds the permissible time interval, bedding mortar must be laid out before construction of the next layer.

\subsection{Mathematical model}

The mathematical model for placement process simulation is defined by Eqns (1)-(4).

Equation (1) is the objective function of the simulation, where $T$ is the placement unit's simulated duration, $S$ is the set of simulation boundary conditions, $M$ is the set of machinery parameters, $O$ is the set of placement unit construction organizational modes, and $P$ is the set of construction parameters under the real-time monitoring system.

Equation (2) defines the variables used in the objective function, where $S_{s}$ is the shape parameters of the placement unit including the length, width, and height; $S_{c}$ is the current construction state of the placement unit that specifies the currently completed layers and placement units; $M_{d}$ is the dozer parameters including the accessible dozer quantity and configuration such as blade width and working inclination; $M_{r}$ is the roller parameters including the accessible roller quantity and roller drum width; $M_{q}$ is the number of accessible nuclear density gauges; $O_{l}$ is the layer construction modes including horizontal-layer construction and slopping-layer construction, and $O_{p}$ is the inner-layer construction modes including sequence construction and flow-process construction. $v_{p}$ is the paving speed, $v_{r}$ is the rolling speed, $\theta_{r}$ is the rolling deviation angle, $p_{N}$ is the ratio of the area with rolling passes $N$ to the whole layer area, $t_{p, s}(j-1)$ is the start time of the paving of layer $j$, and $t_{r, e}(j)$ is the end time of the rolling of layer $j$. 
Equation (3) defines the state transfer function during the simulation. $T_{i}$ is the simulated placement unit duration at moment $i, \Delta t_{i}$ is the simulation clock step length between moments $i-1$ and $i, L_{i}$ is the serial number of the last completed layer at moment $i$, and $l\left(\Delta t_{i}\right)$ is the number of layers completed during the clock step length.

Equation (4) gives the constraints of the placement simulation. $p_{s}$ is the standard ratio of the rolling area to the layer area, where rolling must be continued until $p_{N} \geq p_{s}$ to ensure the compaction quality. $T_{0}$ is the permissible construction time interval between adjacent layers. To ensure the inter-layer combing quality, $t_{p, s}(j)-t_{p, s}(j-1) \leq T_{0}$ must be satisfied for layer $j$. Otherwise, bedding mortar paving is conducted before the construction of layer $j$, which adds the bedding mortar paving time $T_{c}$ to the inter-layer construction time $T_{\text {inter }} . T_{m}$ is the time of mortar paving that happened before layer one. $T_{c}$ is the time of the cooling pipes layout that happened before layer $j=5 k$, where $k=1,2, \ldots$.

$$
\begin{aligned}
& T=f(S, M, O, P), \\
& \left\{\begin{array}{l}
S=\left[S_{s}, S_{c}\right] \\
M=\left[M_{d}, M_{r}, M_{q}\right] \\
O=\left[O_{l}, O_{p}\right] \\
P=\left[v_{p}, v_{r}, \theta_{r}, p_{N}, t_{p, s}(j), t_{r, e}(j)\right]
\end{array}\right. \\
& \left\{\begin{array}{l}
T_{i}=T_{i-1}+\Delta t_{i} \\
L_{i}=L_{i-1}+l\left(\Delta t_{i}\right)
\end{array}\right) \\
& \left\{\begin{array}{l}
p_{N}>p_{s} \\
t_{p, s}(j)-t_{p, s}(j-1) \leq T_{0} \\
t_{p, s}(j)-t_{p, s}(j-1)>T_{0}, T_{\text {inter }}=T_{\text {inter }}+T_{c} . \\
T_{\text {inter }}=T_{\text {inter }}+T_{m}, j=1 \\
T_{\text {inter }}=T_{\text {inter }}+T_{c}, j=5 k, k=1,2, \ldots
\end{array}\right.
\end{aligned}
$$

\subsection{Simulation procedure}

The procedure of the RCC dam placement process simulation is shown in Figure 2, and the main steps are explained as follows.

\section{(1) Boundary conditions input}

Boundary conditions include the length $L$, width $W$, and height $H$ of the placement unit, the layer thickness $h$, the concrete supply capacity $p_{1}$, the comprehensive placement unit construction intensity $p_{2}$, and the permissible time interval between placing layers of the current season $T_{0}$. This information can be input into the simulation system by manual entry. Additionally, $L, W$, and $H$ can be obtained directly, and $p_{2}$ can be inferred from placement units with similar machinery supplies by a C\# interface connected to the storehouse surface construction monitoring system.

(2) Horizontal or slopping layer determination

For horizontal layer construction, the layer area:

$S_{1}=L W$.

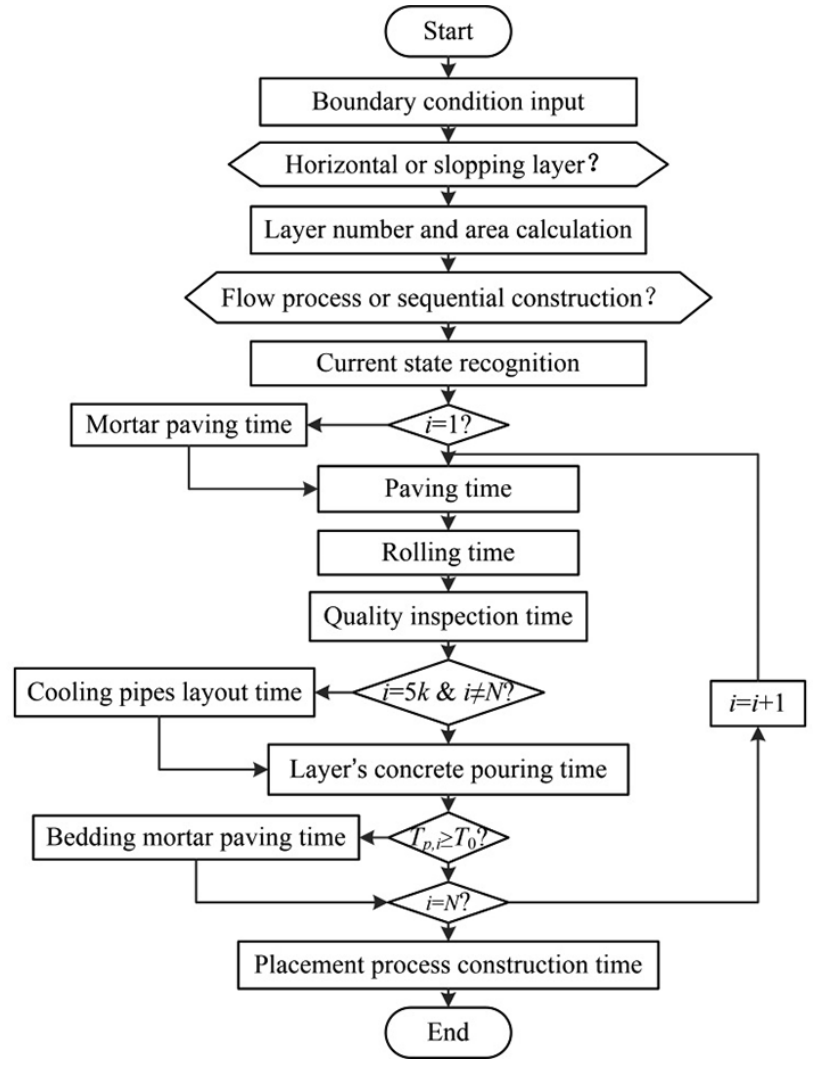

Figure 2. Procedure of RCC dam placement process simulation

For slopping layer construction, the max slopping layer area:

$$
S_{2}=W\left(L_{0}+H / \sin \theta\right)
$$

where $L_{0}$ is the length of initial horizontal segment of slopping layer, $\theta$ is the dig of slopping layer.

Given concrete supply $p_{1}$ and construction intensity $p_{2}$, the maximum constructable area:

$$
S_{\max }=T_{0} \min \left(p_{1}, p_{2}\right) / h .
$$

When $S_{\max } \geq S_{1}$, horizontal-layer construction is adopted; when $S_{2} \leq S_{\max }<S_{1}$, slopping-layer construction is adopted. The partitioning of the slopping layer is shown in Figure 3 . When $S_{\max }<S_{2}$, the concrete supply and construction cannot meet the requirement of the interlayer time interval, and the simulation system will recommend increasing the resource and machinery supply or reducing the placement unit area.

(3) Layer number and area calculation

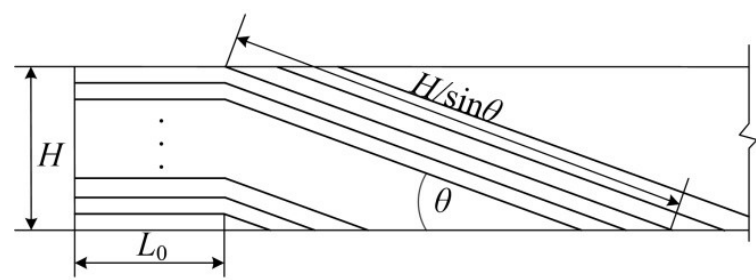

Figure 3. Partition of slopping layers 
For horizontal layer construction, the layer number:

$$
N_{h}=H / h \text {, }
$$

and the layer area:

$$
S_{h}=L W \text {. }
$$

For slopping layer construction, the layer number:

$$
N_{s}=2 H / h+\left(L-2 L_{0}-H \cot \theta\right) \sin \theta / h,
$$

and the corresponding layer area:

$$
S_{s, i}=\left\{\begin{array}{lc}
B\left(L_{0}+i H / \sin \theta\right), & i \leq H / h \\
B H / \sin \theta, \quad H / h<i \leq N_{s}-H / h . \\
B\left(L_{0}+\left(n_{2}-j\right) h / \sin \theta\right), \quad i>N_{s}-H / h
\end{array}\right.
$$

(4) Flow process or sequential construction determination

The construction section width is typically larger than twice the roller drum width or the turning radius of the trucks, i.e. $w=8-12 \mathrm{~m}$. Therefore, the number of dividable construction sections:

$$
m=W / w .
$$

If $m \geq 3$, then flow process construction is adopted; otherwise, sequential construction is adopted.

\section{(5) Current construction state recognition}

Because the placement process simulation may be conducted during construction, recognizing the placement unit $U$ and layer $I$ that are currently under construction is essential for maintaining the construction time calculation. Similar to the boundary conditions input, the state can be either manually input or automatically obtained from the record of the current monitoring layer and section from the real-time monitoring system.

(6) Inner-layer construction time calculation

When flow process construction is adopted because the rolling time of the construction section is larger than the paving time, the inner-layer construction time for the $i$ th layer is:

$$
T_{i}=T_{p 1}+\sum_{j=1}^{m} T_{r j}+T_{q l},
$$

where: $T_{p 1}$ is the paving time of first construction section, $T_{r j}$ is the rolling time of the $j$ th construction section, $T_{q l}$ is the quality inspection time of the last construction section.

The inner-layer construction time under sequential construction is:

$$
T_{i}=T_{p}+T_{r}+T_{q}
$$

where: $T_{p}$ is the paving time of the layer, $T_{r}$ is the rolling time of the layer, and $T_{q}$ is the quality inspection time of the layer.

(7) Inter-layer construction time calculation

Inter-layer construction time:

$$
T_{\text {inter }}=T_{m}+\sum T_{c, i}+\sum T_{u, i}
$$

where $T_{m}$ is the time of cement mortar paving, which only take places before the construction of the first layer; $T_{c, i}$ is the time of the cooling pipe layout, which takes place between layers $i$ and $i+1$; and $T_{u, i}$ is the time of the bedding mortar paving, which takes place when the duration between the start time of paving of the ith layer and the start time of paving of the $i$-1th layer is larger than $T_{0}$.

(8) Placement unit construction time calculation

The placement unit construction time:

$$
T=\sum T_{i}+T_{\text {inter }}, i=I, I+1, \ldots,
$$

where $I$ is the number of the layer currently under construction.

According to this procedure, the CYCLONE model of the placement process is built for conducting simulations, as shown in Figure 4.

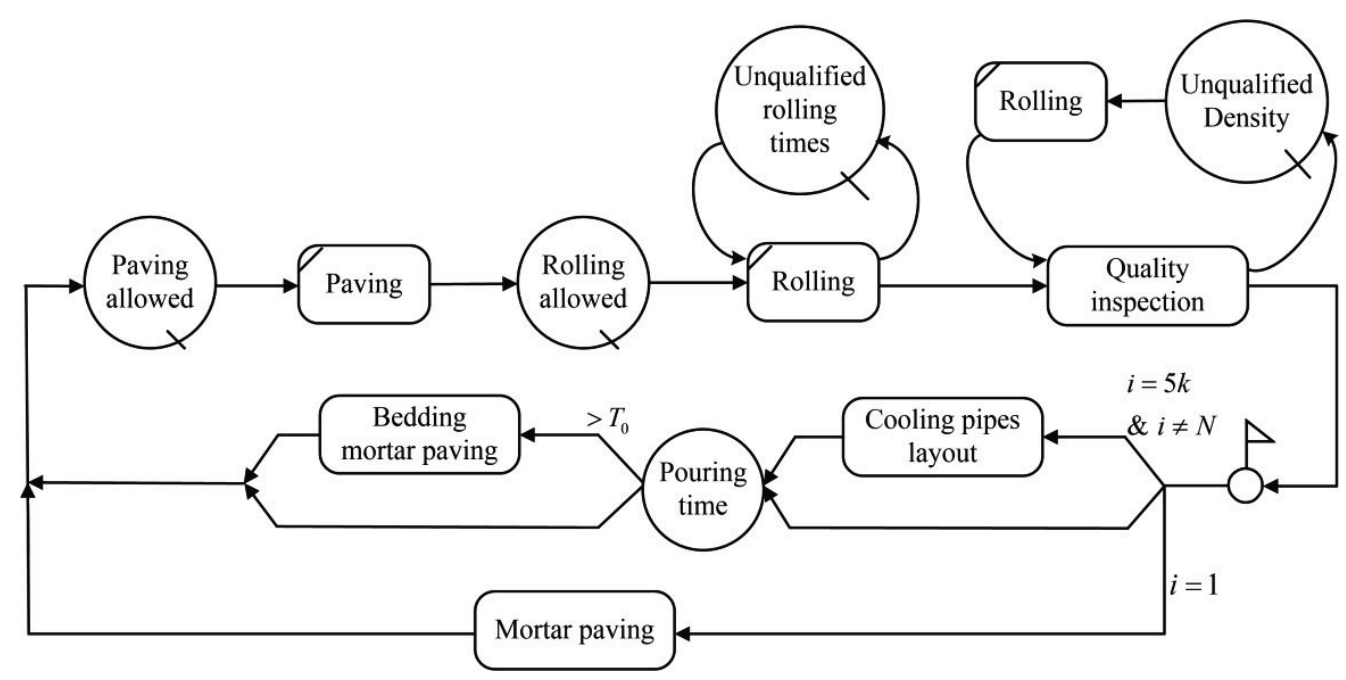

Figure 4. CYCLONE model of placement process 


\section{Construction activity modeling based on real-time monitoring}

\subsection{Background of RCC dam placement unit construction monitoring}

Liu et al. (2015) proposed the real-time monitoring method for RCC dam storehouse surface construction quality control. Real-time monitoring also provides a convenient method for construction data online collection. The key of storehouse surface construction real-time monitoring is to control the compaction quality of the RCC by monitoring rolling process parameters such as the rolling speed, rolling passes, rolling thickness, and vibration status. Dozers are also monitored to control the paving thickness in real time. Additionally, for inter-layer combining quality control, the dozers' start working time and end working time for each layer coupled with the working time of the rollers are used for real-time analysis of the inter-layer construction time. When the parameters exceed the preset quality criteria or the inter-layer construction time approaches the permissible time interval, alarm information will be automatically sent to the site personnel to adjust the construction. Graphical monitoring reports can be produced to serve as supporting files for quality assessment.

The above functions are realized based on real-time construction data collection and transmission. All dozers and rollers are equipped with high-precision satellite positioning devices and supported by a real-time kinematic (RTK) technique to acquire positioning data including the latitude and longitude coordinates, altitude, deviation angel, and velocity. Vibration status monitoring units (VSMU) that judge the vibration status by high- or low-voltage signals are also equipped on the rollers. Data transfer units (DTU) are equipped on every dozer or roller, which transfer the collected positioning and vibration status data to the center server through the GPRS network. The frequency of the above data collection and transmission is $1 \mathrm{~Hz}$. The server calculates and analyzes parameters in real time based on the collected data and visualizes the information on a client program. For details on RCC dam storehouse surface construction real-time monitoring technology, please refer to Liu et al. (2015).

\subsection{Inner-layer construction activities modeling}

\subsubsection{Paving}

Paving activity is a random process influenced by the discharge point position, paving path, blade inclination, etc. It is difficult to model the paving directly. Therefore, the empirical paving efficiency formula according to the "Design guide of construction equipment selection for hydropower and water conservancy project" (National Economic and Trade Commission of the People's Republic of China, 2001) is used:

$$
\left\{\begin{array}{l}
P=V K_{p} K_{s} K_{t} / t \\
V=H^{2} L \cot \varphi / 2, \\
t=2 l / v+t_{0}
\end{array}\right.
$$

where: $H$ is dozer blade width, $H=3.338 \mathrm{~m} ; L$ is dozer blade height, $L=1.149 \mathrm{~m} ; \varphi$ is the natural inclination of RCC during paving, $\varphi=25^{\circ} ; K_{p}$ is the slope coefficient, $K_{p}=1.0 ; K_{s}$ is the loss coefficient, $K_{s}=0.95 ; K_{t}$ is the time utilization coefficient, $K_{t}=0.75 ; t$ is single paving circle time; $v$ is the paving speed; $t_{0}$ is shifting gear time when changing the paving direction, $t_{0}=60 \mathrm{~s}$.

During the simulation, $v$ is randomly generated according to the distribution of paving speed and paving efficiency $P$ is calculated. The layer's concrete volume $T_{p}=V / P$, where $S_{i}$ is layer's area according to Eqn (9) or (11). And the paving time is $T_{p}=V / P$.

\subsubsection{Rolling}

The rolling procedure is the most critical element of the construction process and not only influences the density, smoothness, and surface texture but also has the longest duration among the construction activities. To accurately characterize the rolling's impact on the schedule; a discrete time model is applied to simulate the rolling process.

During the rolling process, the roller reciprocates along the direction of the dam axis. The process can be seen as the roller's random walk process under the joint effect of the roller speed $v^{r}$ and the deviation angle $\theta$. As shown in Figure 5, based on the probability distributions inferred from real-time monitoring data, $v_{t}^{r}$ and $\theta_{t}$ are randomly

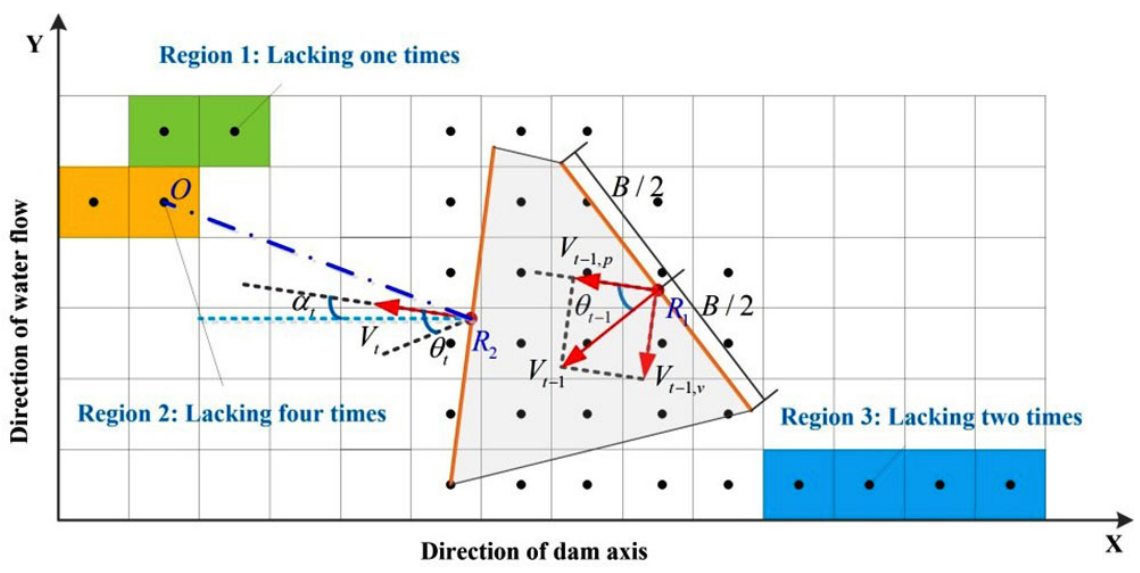

Figure 5. Random walk process and rolling times calculation 
generated each second to simulate the roller's movement as follows:

$$
\left\{\begin{array}{l}
x_{t}=x_{t-1}+\frac{1}{2}\left(v_{t-1, p}^{r}+v_{t}^{r}\right) \Delta t \cos \alpha_{t}+\frac{1}{2} v_{t-1, p}^{r} \Delta t \sin \alpha_{t} \\
y_{t}=y_{t-1}+\frac{1}{2}\left(v_{t-1, p}^{r}+v_{t}^{r}\right) \Delta t \sin \alpha_{t}+\frac{1}{2} v_{t-1, p}^{r} \Delta t \cos \alpha_{t} \\
\alpha_{t}=\sum_{i=0}^{t} \theta_{i}
\end{array}\right.
$$

where: $x_{t}$ and $y_{t}$ are the roller's coordinates at time $t$; $v_{t}^{r}$ is the random roller speed at time $t ; v_{t-1, p}^{r}$ is the $v_{t-1}^{r}$ component in the direction of deviation at time $t-1$, where $v_{t-1, p}^{r}=v_{t-1}^{r} \cos \theta_{t-1} ; v_{t-1, v}^{r}$ is the $v_{t-1}^{r}$ component in the vertical direction of deviation at time $t-1$, where $v_{t-1, p}^{r}=v_{t-1}^{r} \sin \theta_{t-1} ; \alpha_{t}$ is the angle between the direction of $v_{t}^{r}$ and the dam axis; and $\Delta t$ is the fixed time interval, taken as one second.

When the roller reaches the boundary of the water flow direction, one is added to the rolling times of the current band. If the strip reaches the standard rolling time, the roller turns to the next strip with the maximum deviation angle $\theta_{\max }$ and a random $v_{r}^{i}$. Like the $2 \#$ roller shown in Figure 6, when $y_{t} \leq Y_{m}$ is satisfied, the roller's transfer from strip $i-1$ to strip $i$ is finished. Then, the roller starts the random movement at strip $i$ with newly generated $v_{r, t}$ and $\theta_{t}$.

When all the strips reach the standard rolling time, the first rolling time is finished. However, due to the random movement of the rollers, not everywhere within the rolling area reaches the standard rolling time $N_{S}$. Rollers will be arranged to give additional rolling to these areas lacking rolling, which costs additional rolling time in the straight schedule line. To measure this additional remedial rolling time, the rolling area is partitioned into several one-meter by one-meter grids, and each grid's rolling time is calculated during the simulation.

Taking $\left(x_{t-1}, y_{t-1}\right)$ and $\left(x_{t}, y_{t}\right)$ as the midpoints, two lines with lengths equal to the roller drum width can be drawn in the vertical direction of the corresponding rolling speeds. A quadrilateral reflecting the effective rolling area can be obtained by connecting the line end points. The grids under the whole cover of the quadrilateral are regarded as rolled once, whose rolling times add one. For grids that are partly covered, only those whose center point is covered are regarded as rolled one more time.
After the first rolling time, the rolling area standard rolling time ratio $p_{N}$ is calculated as:

$$
p_{N}=\frac{e_{\geq N}}{e_{G}}
$$

where $e_{\geq N}$ is the number of grid sections with rolling times equal to or larger than $N$ and $e_{G}$ is the number of grid sections in the whole rolling area.

Under the actual real time-monitored rolling process, if $p_{N}<95 \%$, additional rolling is conducted on the regions lacking rolling. In the simulation, grid sections with less rolling time get additional rolling with priority. As shown in Figure 5, additional rolling will be conducted on Region 2, Region 3, and Region 1 in turn. Upon heading to the region lacking rolling, the roller takes a random $v_{t}^{r}$ along the direction with the shortest path to the grid section's center point, i.e., the direction of $\mathrm{R}_{2} \mathrm{O}$, to Region 2 in Figure 5. Additional rolling is also a random movement with a randomly generated $v_{t}^{r}$ and $\theta_{t}$, such as the first rolling time. $p_{N}$ is calculated again after additional rolling of a region. Once $p_{N} \geq 95 \%$, the additional rolling is completed.

The sum of the first rolling time $T_{r 1}$ and the additional remedial rolling time $T_{r 2}$ is the area's rolling time $T_{r}$.

\subsubsection{Quality inspection}

After rolling of the whole layer under horizontal-layer construction or of the construction section under slopping-layer construction, quality inspection is conducted to measure the roller-compacted concrete density. The inspection uses a nuclear densimeter at randomly selected measuring points. The number of points is proportional to the area of the layer or section pending testing, typically one point per two hundred square meters.

The method of modeling the quality inspection time is as follows.

The layers finished without cooling pipe layout or bedding mortar paving, i.e., those with $i \neq n k, n=5, k=1,2, \ldots$ and $t_{i}<T_{0}$, are selected before the next layer's construction. The inspection time is:

$$
t_{q}=t_{i+1, p s}-t_{i, r e}
$$

where $t_{i+1, p s}$ is the start time of the paving of layer $i+1$ and $t_{i, r e}$ is the ending time of the rolling of layer $i$.

Meanwhile, the inspection region area $S$ is obtained, which is used to calculate the number of measuring points,

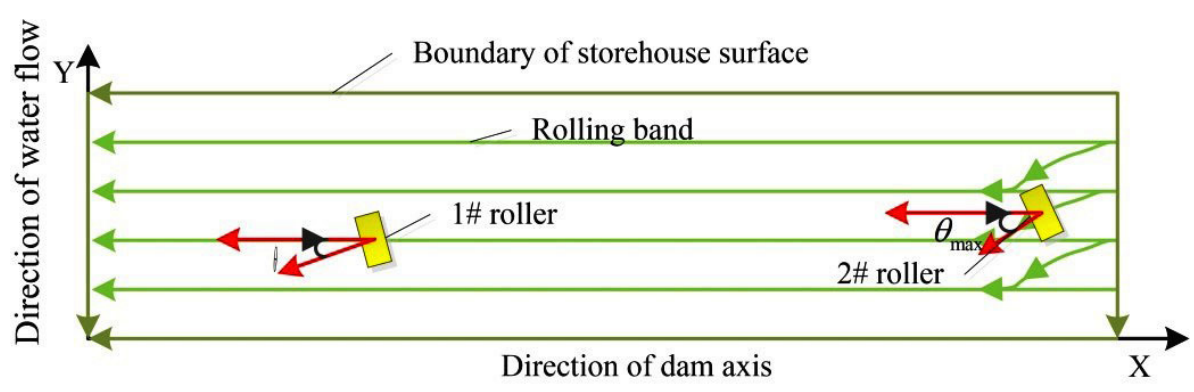

Figure 6. Rolling bands and rolling deviation 


$$
n=\left\{\begin{array}{l}
{[k]+1,[k] \geq 0.5} \\
{[k],[k]<0.5}
\end{array},\right.
$$

where $k=S / 200$ and $[k]$ represents the maximum integer that is not larger than $k$.

So the single point inspection time is:

$$
t_{q 0}=t_{q} / n \text {. }
$$

According to the above procedure, the samples of $t_{q 0}$ can be obtained, and its distribution can be inferred. In the simulation, the measuring point number $n$ is first calculated using Eqn (21). Then, $n$ single-point inspection times are randomly generated according to $t_{q 0}$ 's distribution, whose sum corresponds to $T_{q i}$ or $T_{q l i}$.

\subsection{Inter-layer construction activities modeling}

\subsubsection{Mortar paving}

The mortar paving time of a placement unit is

$$
t_{m}=t_{1, p s}-t_{s}
$$

where $t_{s}$ is the start time of monitoring of the placement unit and $t_{1, p s}$ is the start time of paving of the first layer.

Therefore, the mortar paving time per unit area is:

$$
t_{m 0}=t_{m} / S_{1}
$$

where $S_{1}$ is the area of the first layer.

The distribution of $t_{m 0}$ can be inferred based on samples from the real-time monitoring system.

In the simulation, when $i=1$, a random mortar paving time per unit area is generated, which is multiplied by $S_{1}$ to yield $T_{m}$.

\subsubsection{Cooling pipes layout}

The layers with serial number that satisfies $i=n k, n=5, k=1,2, \ldots, \quad i \neq N$ and $T_{i}<T_{0}$ are picked out. The cooling pipes layout time is:

$$
t_{c}=t_{i+1, p s}-t_{i, r e}-T_{q},
$$

where $T_{q}$ is $T_{q i}$ under horizontal layer construction or $T_{q l i}$ under slopping layer construction.

So the cooling pipes layout per unit area is:

$$
t_{c 0}=t_{c} / S_{i}
$$

where $S_{i}$ is the area of layer $i$.

The distribution of $t_{c 0}$ can be inferred based on samples from real-time monitoring system.

In simulation, when $i \neq n k, n=5, k=1,2, \ldots$ and $i \neq N$, a random cooling pipes layout time per unit area is generated, which multiplied by $S_{i}$ to yield $T_{c, i}$.

\subsubsection{Bedding mortar paving}

For the layers with $i \neq N$, when the layer concrete pouring time $T_{p, i}$ exceeds the permissible time interval $T_{0}$, where $T_{p, i}=\left\{\begin{array}{l}T_{i}, \quad i \neq n k, n=5, k=1,2, \ldots \\ T_{i}+T_{c, i}, \text { otherwise }\end{array}\right.$,

the bedding mortar paving is conducted to guarantee joint quality before the next layer's construction. To avoid cooling pipes layout time's interference, only the layers with $T_{p, i} \geq T_{0}$ and $i \neq n k, n=5, k=1,2, \ldots$ are picked out. The bedding mortar paving time is:

$$
t_{u}=t_{i+1, p s}-t_{i, r e}-T_{q} .
$$

The bedding mortar paving per unit area is:

$$
t_{u 0}=t_{c} / S_{i} \text {. }
$$

The distribution of $t_{u 0}$ can be inferred based on samples from real-time monitoring system.

In the simulation, when the layer concrete pouring time $T_{p, i}$ is larger than $T_{0}$, a random mortar paving time per unit area is generated, which is multiplied by $S_{j}$ to yield $T_{u, j}$.

\section{Fast DPM models inference for simulation parameter online update}

Simulation is a computer-based sampling process, so the sampling basis, simulation parameters' distributions, directly influences the accuracy of schedule prediction results. Typically, distributions are estimated by expert experience or similar project analogy, which is adaptable for overall construction schedule prediction of planning or design phase. However, construction process, especially like RCC dam construction placement process, is a dynamic resource organization process which is subject to various internal and external environmental factors. This dynamic leads the simulation parameters' distribution to change over time. To model the actual construction more accurately, Dirichlet process mixture (DPM) model is applied to estimate the simulation parameters' distribution based on real-time data.

In previous researches on construction simulation, maximum likelihood estimation and Bayesian parameter estimation are applied to simulation parameter update. Both methods constrain the inference to a specific parametric form, which may limit the scope and inferences types. However, actual parameters' distribution is dynamic and complex, calling for a more robust and flexible modeling method.

DPM models, a class of nonparametric Bayesian models, are concerned with inference for unknown density at infinite dimensional parameter space. Due to the ability to give more details about complex density apart from low dimensional summaries like means, DPM models have been used in density estimation problems in signal process and biostatistics (Rabaoui, Viandier, Duflos, Marais, \& Vanheeghe, 2012; Pereira, Ferré, Giremus, \& Grivel, 2014; Lennox, Dahl, Vannucci, Day, \& Tsai, 2010; Zhao, Kang, \& Yu, 2014). In this paper, DPM models are used to estimate simulation parameters probability density. 
Besides, considering dynamic simulation based on realtime monitoring is a continuous data streaming application, a fast DPM models inference algorithm is proposed.

\subsection{DPM models for density estimation}

Suppose $\boldsymbol{x}^{(n)}=\left(x_{1}, \ldots, x_{n}\right)$ are a set of observed independent identically distributed samples drawn from an unknown distribution $F$. A mixture model to estimate $F$ on basis of $\boldsymbol{x}^{(n)}$ is given by:

$$
F(x)=\int_{\Theta} f(x \mid \theta) d G(\theta),
$$

where: $\theta \in \Theta$ is called latent variable, $f(\cdot \mid \theta)$ is the mixed density, $G$ is the mixing distribution.

When $G$ is assumed as a random probability measure with a Dirichlet process (DP) prior:

$$
G \sim \operatorname{DP}\left(\alpha, G_{0}\right),
$$

Eqns (30) and (31) together form the DPM models, where $\alpha$ is the precision parameter and $G_{0}$ is the base distribution.

DPM models can be rewritten as a hierarchical form:

$$
\left\{\begin{array}{l}
x_{i} \mid \theta_{i} \sim f\left(x \mid \theta_{i}\right) \\
\theta_{i} \sim G \\
G \sim D P\left(\alpha, G_{0}\right)
\end{array},\right.
$$

where $\theta_{i}$ is the distribution parameter specific to observation $x_{i}$. Normal kernel $f\left(\cdot \mid \theta_{i}\right)=N\left(\cdot \mid \mu_{i}, \sigma_{i}\right)$ is used in this paper, with $\theta_{i}=\left(\mu_{i}, \sigma_{i}\right)$. Figure 7 explains this hierarchical model structure. Details of DPM models can refer to Müller, Quintana, Jara, and Hanson (2015).

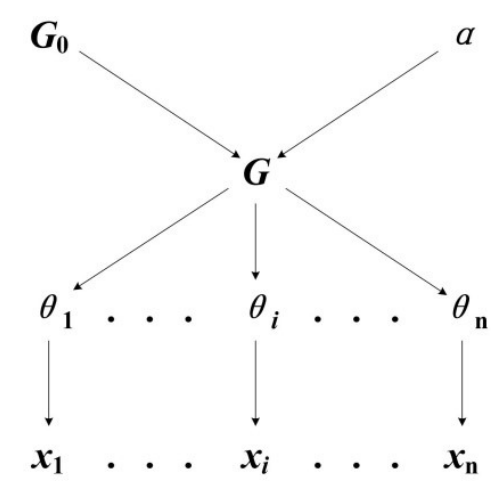

Figure 7. Hierarchical structure of DPM models

\subsection{Improved SUGS algorithm for fast inference}

Currently, Markov Chain Monte Carlo (MCMC) based algorithms are widely investigated to compute DPM models' posterior. However, DPM models' advantage of incorporating uncertainty at the level of distribution functions also brings great computational cost. Processing a moderate sized dataset can even cost several hours. To copy with real-time streaming data, it is critical to propose a fast inference algorithm. Sequential updating and greedy search (SUGS) algorithm was proposed by Wang and Dunson
(2011) for posterior computation and model selection in Dirichlet process mixture models, which was proved fast and can be implemented easily in large datasets. An improved SUGS algorithm is proposed for simulation parameter density estimation.

\subsubsection{Procedure of improved SUGS}

The core of SUGS algorithm is allocating observations to classes according to maximizing the conditional posterior probability. Let $x_{i}$ denote the observation and $\gamma_{i}$ denote the class label of the $i$ th observation. The available information at time $n$ are $\boldsymbol{x}^{(n)}=\left\{x_{1}, \ldots, x_{n}\right\}$ and $\gamma^{(n-1)}=\left\{\gamma_{1}, \ldots, \gamma_{n-1}\right\}$. The core of SUGS algorithm is to choose $\gamma_{i}$ to maximize the conditional probability of $\gamma_{i}=h$ given $\boldsymbol{x}^{(i)}$ and $\boldsymbol{\gamma}^{(i-1)}$ :

$$
\gamma_{i}=\arg \max _{1 \leq h \leq k_{i-1}+1} P\left(\gamma_{i}=h \mid \boldsymbol{x}^{(i)}, \boldsymbol{\gamma}^{(i-1)}\right),
$$

and to update the posterior distribution using $x_{i}$ and $\gamma_{i}$ :

$$
\pi\left(\theta_{\gamma_{i}} \mid \boldsymbol{x}^{(i)}, \boldsymbol{\gamma}^{(i)}\right) \propto f\left(x_{i} \mid \theta_{\gamma_{i}}\right) \pi\left(\theta_{\gamma_{i}} \mid \boldsymbol{x}^{(i-1)}, \boldsymbol{\gamma}^{(i-1)}\right),
$$

where $\theta_{h}$ are the parameters of class $h, f\left(x_{i} \mid \theta_{\gamma_{i}}\right)$ is the observation density conditioned on class $\gamma_{i}, k_{i-1}$ is the number of classes at time $i-1$.

The above procedure relies on the ordering of data. Original SUGS suggested to apply the algorithm to several random reordered sequences of $\boldsymbol{x}^{(n)}$ and choose the sequence with maximum pseudo-marginal likelihood for inference. However, there are multiple reordered sequences especially when dealing with large amount data. Limited number of random produced sequences may lose some features of the whole sequence population. To improve the representative of the sequence samples, permutation entropy $(P E)$ is applied here to evaluate the randomness of sequences and assure the sequences with different level of randomness are picked out for pseudo-marginal likelihood comparison.

Permutation entropy $(P E)$ was proposed by Bandt and Pompe (2002) to measure the randomness of one-dimensional time series.

Unlike previous entropy approaches, $P E$ has the advantages of robustness, lower computational requirements and easy calculation for chaotic and noisy time series.

Given a certain ordering sequence $\boldsymbol{x}^{(n)}=\left\{x_{1}, \ldots, x_{n}\right\}$, it can be embedded to a $D$-dimensional space $\left\{x(t)=\left[x_{t}, x_{t+\tau}, \ldots, x_{t+(D-1) \tau}\right], t=1,2, \ldots, n-(D-1) \tau\right\}$, where $D$ is the embedding dimension and $\tau$ is the time delay. Each vector $x(t)$ is rearranged in an ascending order as $x(t)=\left[x_{t+\left(i_{1}-1\right) \tau} \leq x_{t+\left(i_{2}-1\right) \tau} \leq \ldots \leq x_{t+\left(i_{D}-1\right) \tau}\right]$. When $x_{t+\left(i_{m}-1\right) \tau}=x_{t+\left(i_{n}-1\right) \tau}$, the order is decided by $m$ and $n$, i.e. if $m<n, x_{t+\left(i_{m}-1\right) \tau}$ is ranked before $x_{t+\left(i_{n}-1\right) \tau}$. Then each $x(t)$ is mapped onto an indice sequence $\left(i_{1}, i_{2}, \ldots, i_{D}\right)$. With a dimension of $D$, there are $D$ ! permutations of indice sequence. The (normalized) permutation entropy of $\boldsymbol{x}^{(n)}$ is calculated as:

$$
P E=-\frac{1}{\ln D !} \sum_{j=1}^{D !} \pi_{j} \ln \pi_{j},
$$


where $D$ ! represents all the possible indice permutations, $\pi_{j}$ represents the frequency of appearance of the $j$-th indice permutation.

The value of $P E, 0 \leq P E \leq 1$, reflects randomness of $\boldsymbol{x}^{(n)}$. The closer $P E$ is to one, the more random is the sequence. And the sequence with $P E$ close to zero is nearly monotonously increasing or decreasing, which has limited dynamics.

At time $n$, to investigate the distribution, $\boldsymbol{x}^{(n)}=\left\{x_{1}, \ldots, x_{n}\right\} \quad$ is reordered as $\boldsymbol{x}_{1}^{(n)}, \boldsymbol{x}_{2}{ }^{(n)}, \ldots$. For $i=1,2, \ldots, P E$ of $\boldsymbol{x}^{(n)}$ is calculated. Considering that the larger randomness means more kinds of ordered sequence, for $(k-1) / S \leq P E \leq k / S, k=1,2, . ., S$, the first $k^{2}$ sequences are picked out. Then these chosen sequences are used for maximum pseudo-marginal likelihood comparison. The maximum pseudo-marginal likelihood is calculated as:

$$
\begin{aligned}
P M L\left(\boldsymbol{x}_{j}^{(n)}\right)= & \prod_{i=1}^{n} \pi\left(x_{i} \mid \boldsymbol{x}^{(-i)}, \boldsymbol{\gamma}^{(-i)}\right)= \\
& \prod_{i=1}^{n} \int\left(\boldsymbol{x}_{i} \mid \boldsymbol{\theta}\right) \pi\left(\boldsymbol{\theta} \mid \boldsymbol{x}^{(-i)}, \boldsymbol{\gamma}^{(-i)}\right) d \boldsymbol{\theta} .
\end{aligned}
$$

Replacing $\pi\left(\boldsymbol{\theta} \mid \boldsymbol{x}^{(-i)}, \boldsymbol{\gamma}^{(-i)}\right)$ with $\pi(\boldsymbol{\theta} \mid \boldsymbol{x}, \boldsymbol{\gamma})$ in the large sample situation, maximum pseudo-marginal likelihood is a product of predictive densities:

$$
\operatorname{PML}\left(\boldsymbol{x}_{j}^{(n)}\right)=\prod_{i=1}^{n} \int \pi\left(x_{i} \mid \boldsymbol{\theta}\right) \pi(\boldsymbol{\theta} \mid \boldsymbol{x}, \boldsymbol{\gamma}) d \boldsymbol{\theta}=\prod_{i=1}^{n} g\left(x_{i}\right),
$$

where $g\left(x_{i}\right)$ is predictive density for upcoming observation $x_{i}$.

The sequence with maximum $P M L$ is used to calculate the predictive density. The predictive density for a new object $x_{n+1}$ is:

$$
\begin{aligned}
g\left(x_{n+1}\right)= & \sum_{h=1}^{k_{n}+1} \boldsymbol{\pi}_{n+1, h} \int f\left(x_{n+1} \mid \boldsymbol{\gamma}_{n+1}=\right. \\
& \left.h \boldsymbol{\theta}_{h}\right) d \boldsymbol{\pi}\left(\boldsymbol{\theta}_{h} \mid \boldsymbol{x}^{(n)}, \boldsymbol{\gamma}^{(n)}\right)= \\
& \sum_{h=1}^{k_{n}+1} \boldsymbol{\pi}_{n+1, h} \int f\left(x_{n+1} \mid \boldsymbol{\gamma}_{n+1}=\right. \\
& \left.h, \boldsymbol{x}^{(n)}, \boldsymbol{\gamma}^{(n)}\right),
\end{aligned}
$$

where $x^{(-i)}$ is the set of observations except of $x_{i}, \gamma^{(-i)}$ is the set of class labels except of $\gamma_{i}$.

To accelerate computation, $\pi\left(\boldsymbol{\theta} \mid \boldsymbol{x}^{(-i)}, \boldsymbol{\gamma}^{(-i)}\right)$ is replaced with $\pi(\boldsymbol{\theta} \mid \boldsymbol{x}, \boldsymbol{\gamma})$ which is accurate particularly for large samples.

The pseudo code for the improved SUGS is given in Table 1.

\subsubsection{Model parameters update}

\section{(1) Concentration Parameter $\alpha$ update}

DPM precision parameter $\alpha$ is critical, because it directly influences the allocation of subjects to clusters. Tsiligkaridis and Forsythe (2015) proposed a data-driven
Table 1. Improved SUGS algorithm

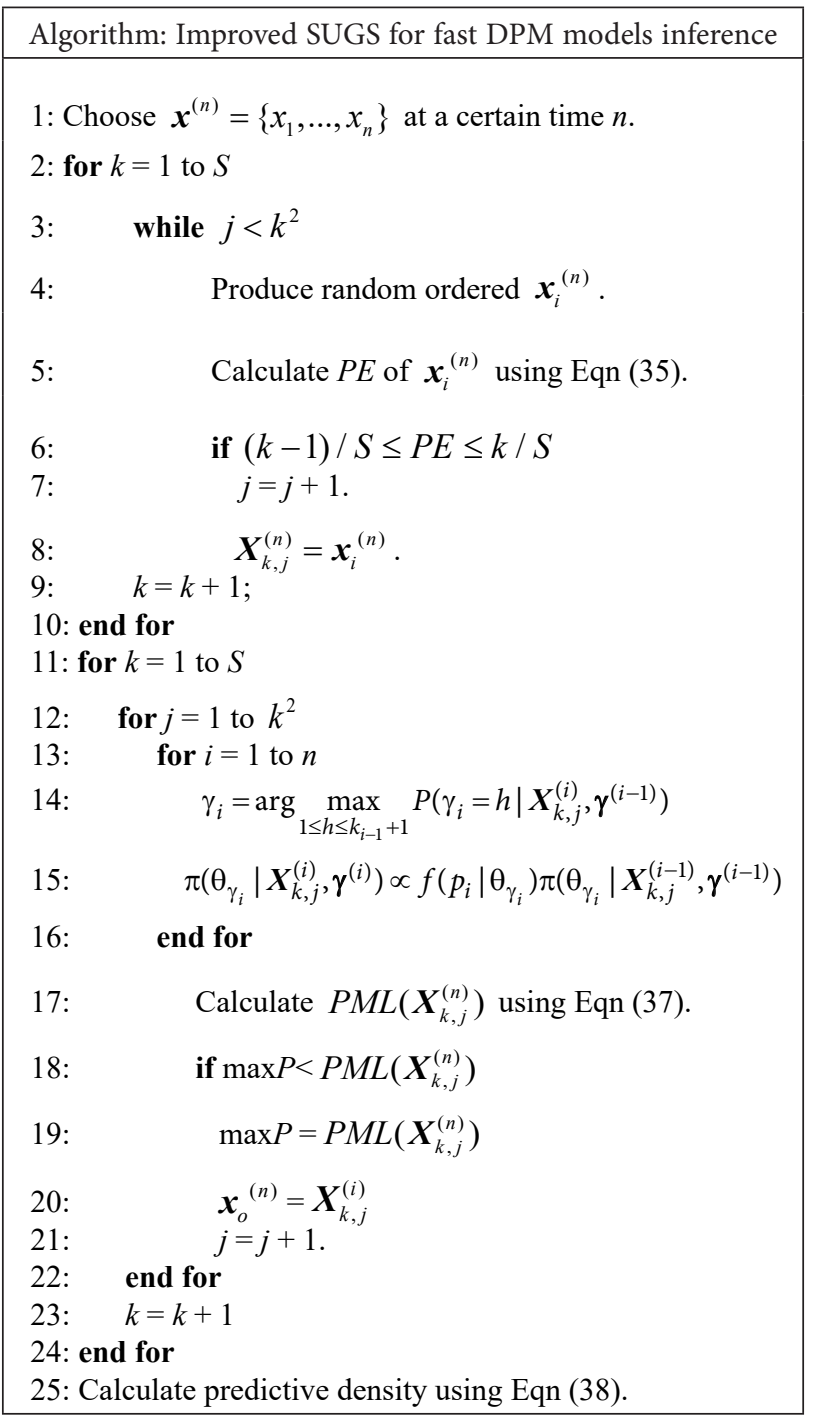

method to adaptive update $\alpha$ with newly obtained data. A prior distribution $p_{1}(\alpha)$ is assigned to $\alpha$, and the distribution of $\alpha$ is adaptively updated according to

$$
\begin{aligned}
& p_{i}(\alpha) \propto p\left(x_{i}, \gamma_{i} \mid x^{(i-1)}, \gamma^{(i-1)}, \alpha\right) p\left(\alpha \mid x^{(i-1)}, \gamma^{(i-1)}\right) \propto \\
& p_{i-1}(\alpha) \pi_{i, \gamma_{i}}(\alpha), \quad i=2,3, \ldots,
\end{aligned}
$$

where $p_{i}(\alpha)=p\left(\alpha \mid x^{(i)}, \gamma^{(i)}\right)$ denote the posterior distribution for $\alpha$ at time $i$, and $\alpha$ that used is the mean of distribution $p_{i-1}(\alpha)$.

Tsiligkaridis and Forsythe (2015) further demonstrate that the distribution $p_{i}(\alpha)$ can be approximated by a Gamma distribution with shape parameter $k_{i}$ and rate parameter $\lambda+\log i$, where $\alpha_{i}=\frac{k_{i}}{\lambda+\log i}$ only relies on
update of $k_{i}$ at iteration $i$.

(2) Mixture parameters update

Normal mixture model is typically used in DPM analysis. Normal model with parameter $\theta_{h}=\left(\mu_{h}, \tau_{h}\right)^{\prime}$, where $\mu_{h}$ is mean and $\tau_{h}$ is variance, is assigned to cluster $h$, 
$h=1, \ldots, \infty$. To speed up computation, normal distribution's conjugate distribution, normal-Gamma distribution is used as prior for $\theta_{h}$ :

$$
\pi\left(\mu_{h}, \tau_{h}\right)=N_{p}\left(\mu_{h} \mid m_{0},\left(\psi_{0} \tau_{h}\right)^{-1}\right) \operatorname{Gamma}\left(\tau_{h} \mid a_{0}, b_{0}\right),
$$

where $m, \psi, a, b$ are hyperparameters that assumed known.

As observations $\boldsymbol{x}^{(i)}$ and partitions $\boldsymbol{\gamma}^{(i)}$ continuously obtained, it is convenient to update the prior as follows:

$$
\begin{aligned}
\pi\left(\mu_{h}, \tau_{h} \mid \boldsymbol{x}^{(i)}, \boldsymbol{\gamma}^{(i)}\right)= & N_{p}\left(\mu_{h} \mid m_{h}^{(i)},\left(\psi_{h}^{(i)} \tau_{h}\right)^{-1}\right) \\
& \operatorname{Gamma}\left(\tau_{h} \mid a_{h}^{(i)}, b_{h}^{(i)}\right),
\end{aligned}
$$

where hyperparameters are calculated as follows:

$$
\left\{\begin{array}{l}
\psi_{h}^{(i)}=\psi_{h}^{(i-1)}+1 \\
m_{h}^{(i)}=\left(\psi_{h}^{(i-1)} m_{h}^{(i-1)}+x_{i}\right) / \psi_{h}^{(i)} \\
a_{h}^{(i)}=a_{h}^{(i-1)}+1 / 2 \\
b_{h}^{(i)}=b_{h}^{(i-1)}+\frac{\psi_{h}^{(i-1)}\left(x_{i}-m_{h}^{(i-1)}\right)^{2}}{2\left(\psi_{h}^{(i-1)}+1\right)}-b^{(i-1)}+b^{(i)}
\end{array},\right.
$$

where $m_{h}^{(0)}=0, \psi_{0}=1, a_{h}^{(0)}=1, \pi\left(b_{0}\right)=\operatorname{Gamma}(1,10)$ is recommended after normalizing the data (Wang \& Dunson, 2011).

\section{Case study}

HD dam, shown in Figure 8, is a RCC dam located in Yunnan province, southwest China. The dam, consists of 20 dam sections, is 203 meters tall and 464 meters long. The total dam concrete volume is $350.7 \times 10^{4} \mathrm{~m}^{3}$, of which $261.9 \times 10^{4} \mathrm{~m}^{3}$ is roller compaction concrete. The construction process of HD dam is analyzed to demonstrate the applicability of the proposed method.

\subsection{Proposed improved SUGS algorithm illustration}

The performance of proposed improved SUGS algorithm is tested by comparison with SUGS algorithm, MCMC based DPM models method and kernel density estimation method. A mixture of three normal distributions used by Wang and Dunson (2011) is set as true density:

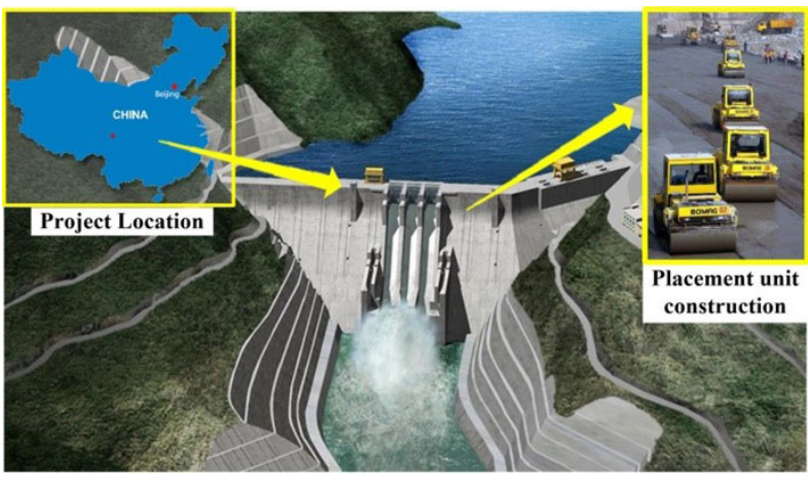

Figure 8. Location of HD RCC dam $f(x)=0.3 N(x ;-2,0.4)+0.5 N(x ; 0,0.3)+0.2 N(x ; 2.5,0.3)$.

There are 100 sets of samples with size $n=100$, $n=200, n=500$ randomly drew from the true density. For each set, predictive density is estimated using the proposed algorithm, SUGS algorithm, MCMC based DPM models method and kernel density estimation method, respectively. Kullback-Leibler divergence $(K L D)$ between true density and estimation is used to measure the closeness of different methods:

$$
K L D(f, g)=\int f(x) \log \left\{\frac{f(x)}{g(x)}\right\} d x,
$$

where $g(x)$ is predictive density.

The proposed algorithm takes the priors as described in section 5.2. The SUGS algorithm uses the same prior except giving $\alpha$ a discretized Gamma $(1,1)$ distribution prior with support on the points $\{0.01,0.05\} \cup\{0.1+0.2 k, k=0,1, \ldots, 20\}$, which is recommended by Wang and Dunson (2011). SUGS algorithm is repeated for 20 random orderings. MCMC based DPM models method is conducted using "DPdensity" of "DPpackage" in R. Kernel density estimation method is conducted using the default function "ksdensity" in matlab. Average KLDs of 100 sets of samples using different algorithms are shown in Table 2.

Table 2. Average KLD of each algorithm

\begin{tabular}{|ccccc|}
\hline Sample size & Proposed & SUGS & MCMC & Kernel \\
\hline 100 & 0.0782 & 0.0864 & 0.0643 & 0.0967 \\
200 & 0.0416 & 0.0907 & 0.0393 & 0.0664 \\
500 & 0.0127 & 0.0111 & 0.0103 & 0.0405 \\
\hline
\end{tabular}

As Table 2 showed, the average KLDs of the proposed algorithm are closest to that of MCMC based method, which are all smaller than kernel density estimation method's. Without time-consuming computing, the proposed method reaches near performance with MCMC based method. For SUGS, it has similar average KLDs with the proposed method when sample size equals 100 or 500 . But when sample size is 200 , the average $K L D$ is relatively larger. This is because SUGS is based on limited random sampled sequences that cannot always guarantee an optimal solution. The proposed method alleviates this by establishing several permutations with different PEs. Compared with the above nonparametric probability density estimation algorithms, it proves that the proposed method is suitable for real-time data based, dynamic and complex simulation parameter probability density estimation.

\subsection{Simulation parameter online update}

The RCC dam is divided into ten construction areas as shown in Figure 9. Each area, covering several dam sections, is further divided into several placement units with similar shape and same machinery supply. Because the 


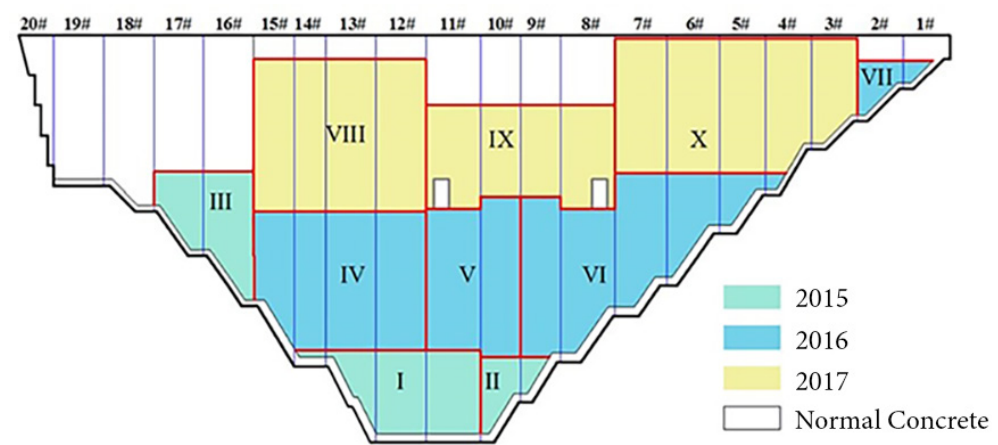

Figure 9. Construction areas of the RCC dam

placement unit's shape and machinery supply directly affected the rolling and paving process, the update of paving speed, rolling speed, and rolling deviation angle are conducted to each construction areas separately. Meanwhile, unit time of quality inspection, mortar paving, cooling pipes layout and bedding mortar paving remains similar among different construction areas, so they are constantly updated since the start of the first placement unit construction.

For paving speed $v^{p}$, rolling speed $v^{r}$, and roller deviation angle $\theta$, their update are based on the real-time monitoring data in each construction area alone. Placement unit (11-13)\# 1426.5-1429.5, with an max construction area of 3895.69 square meters, is the first placement unit under construction in area I. The placement unit adopts horizontal-layer construction method, which consists of 10 layers and is equipped with 3 dozers and 5 rollers. For layer 1, its construction lasted from 6:32 July 3rd, 2015 to 12:32 July 3rd, 2015. Excluding the speed that smaller than $0.15 \mathrm{~km} / \mathrm{h}$ in continuous 5 seconds, 48901 records of rolling speed and 37432 records of paving speed are left for density estimation.

The predictive density of paving speed are shown in Figure 10. It can be seen that paving speed is centered at three peaks around $1.19 \mathrm{~km} / \mathrm{h}, 2.62 \mathrm{~km} / \mathrm{h}$, and $4.43 \mathrm{~km} / \mathrm{h}$. According to the inference result, each peak is corresponding to a cluster. The probability of the next paving speed falls into cluster one with $\pi\left(\mu_{1}, \tau_{1}\right)=$ $N_{p}\left(\mu_{1} \mid-0.8828,\left(0.0044 \tau_{1}\right)^{-1}\right)$ Gamma $\left(\tau_{1} \mid 115.5,13.098\right)$, cluster two with $\pi\left(\mu_{2}, \tau_{2}\right)=N_{p}\left(\mu_{2} \mid 0.1503,\left(0.0073 \tau_{2}\right)^{-1}\right)$ $\operatorname{Gamma}\left(\tau_{2} \mid 69.5,3.8561\right)$, and cluster three with $\pi\left(\mu_{3}, \tau_{3}\right)=N_{p}\left(\mu_{3} \mid 1.3814,\left(0.0075 \tau_{3}\right)^{-1}\right)$ Gamma $\left(\tau_{3} \mid 67.5\right.$, $12.5972)$ are $0.422,0.332$, and 0.246 respectively. For the random paving speed generation in simulation, cluster $i$ is first chosen according to corresponding probabilities, then normal distribution's parameters $\left(\mu_{i}, \tau_{i}\right)$ are randomly generated according to $\pi\left(\mu_{i}, \tau_{i}\right)$, and random paving speed is randomly generated according to normal distribution $N\left(\mu_{i}, \tau_{i}\right)$.

And the predictive density for rolling speed and rolling deviation angle are shown in Figure 11 and Figure 12. The generations of random rolling speed and random rolling deviation angle are similar with random paving speed.

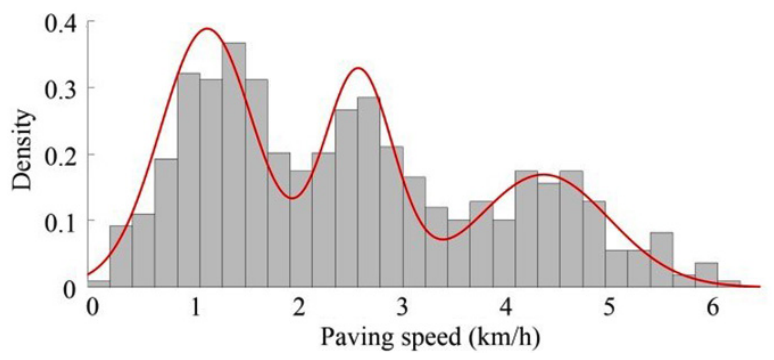

Figure 10. Predictive density of paving speed

Note: The red line represents predictive density function. The histogram is the relative frequency density histogram of paving speed.

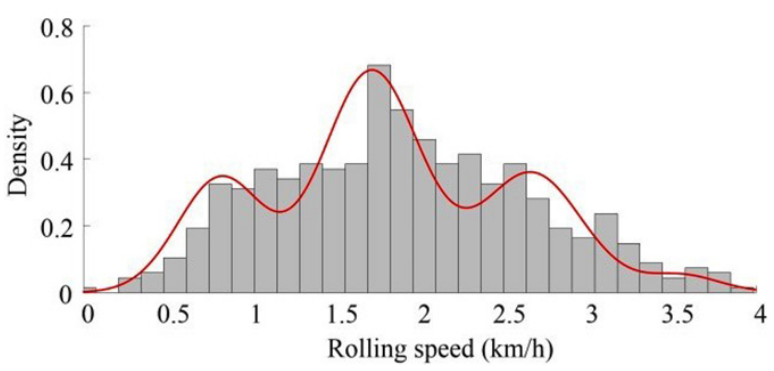

Figure 11. Predictive density of rolling speed

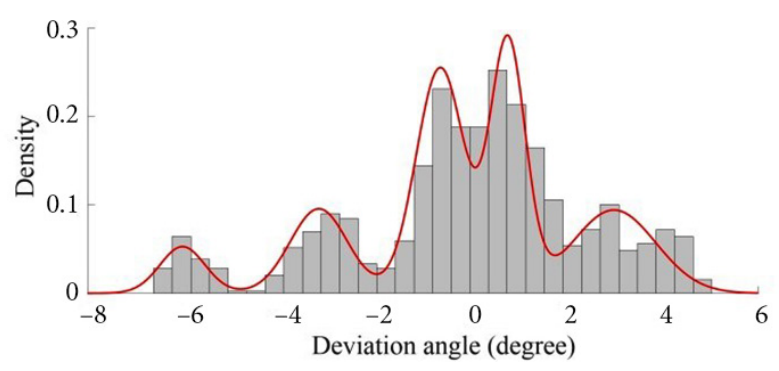

Figure 12. Predictive density of rolling deviation angle

Due to the relative scarcity of data and consistency of operation methods, unit mortar paving time $t_{m 0}$, unit cooling pipes layout time $t_{c 0}$ and unit bedding mortar paving time $t_{u 0}$ are updated during whole RCC placement process, without distinguishing different construction areas. Taking the placement between 15:20 May $18^{\text {th }}$, 


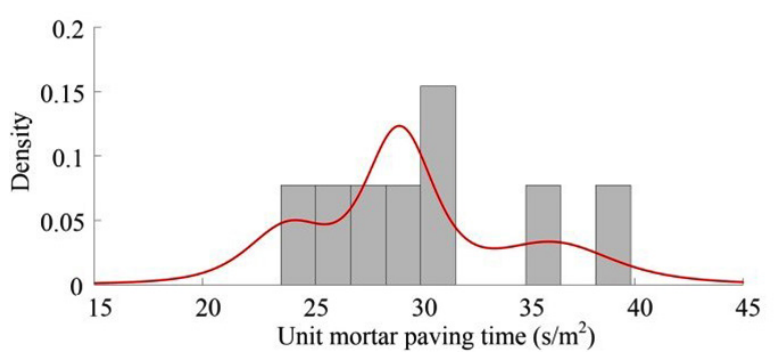

Figure 13. Predictive density of unit mortar paving time

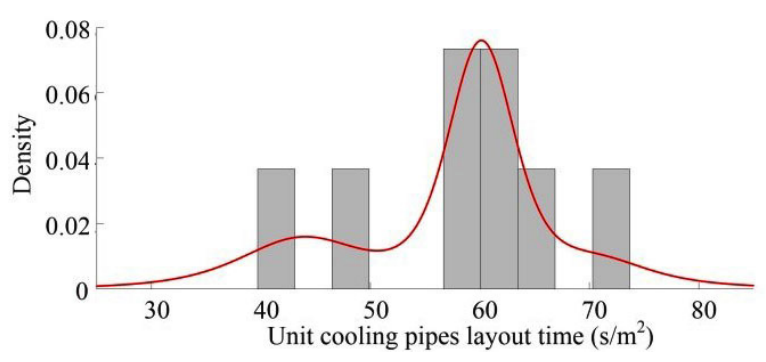

Figure 14. Predictive density of unit cooling pipes layout time

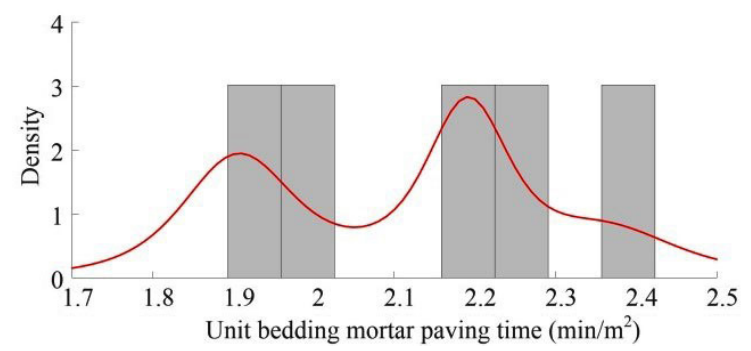

Figure 15. Predictive density of unit bedding mortar paving time

2015 and 8:42 August $15^{\text {th }}, 2015$ as an example, after the construction of four placement unit in each area I and area II, there are eight records of $t_{m 0}$, eight records of $t_{c 0}$, and five records of $t_{u 0}$. The predictive densities of $t_{m 0}, t_{c 0}$, and $t_{u 0}$ are shown in Figure 13, Figure 14, and Figure 15.

To show the dynamic of simulation parameter, rolling speed' density is taken as an example and analyzed at different time. The first, fifth and last placement units in construction area I are picked out and rolling speed density is estimated at end of the first and tenth layer of each placement unit. Figure 16(a) shows that rolling speed of first placement unit is relatively evenly distributed along the interval $[0,4.5 \mathrm{~km} / \mathrm{h}]$ and the interval $[0,5 \mathrm{~km} / \mathrm{h}]$ with three or four peaks. Figure 16(b) shows that rolling speed of fifth placement unit is distributed along the interval $[0$, $4.5 \mathrm{~km} / \mathrm{h}]$ with concentration trends around $[0.75 \mathrm{~km} / \mathrm{h}$, $1.25 \mathrm{~km} / \mathrm{h}]$ and $[1.8 \mathrm{~km} / \mathrm{h}, 3 \mathrm{~km} / \mathrm{h}]$. Figure $16(\mathrm{c})$ shows that rolling speed of last placement unit is distributed along the interval $[0,4 \mathrm{~km} / \mathrm{h}]$ with concentration trends around $[0.75 \mathrm{~km} / \mathrm{h}, 1.5 \mathrm{~km} / \mathrm{h}]$ and [2 km/h, $2.6 \mathrm{~km} / \mathrm{h}]$. Comparing Figures 16(a), 16(b), and 16(c), it's obvious that the distribution of rolling speed is gradually concentrating and the overall rolling speed decrease as the construction (a)

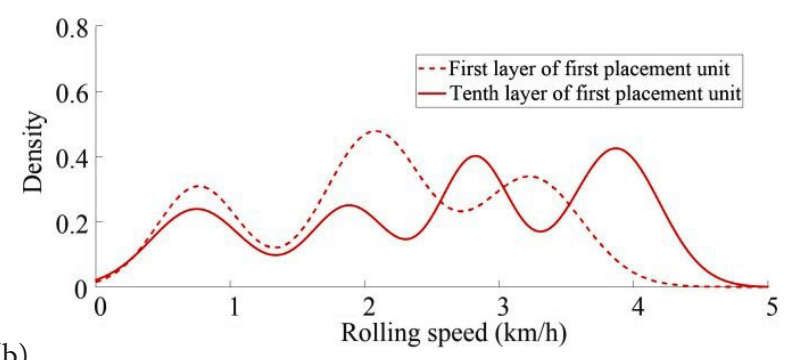

(b)

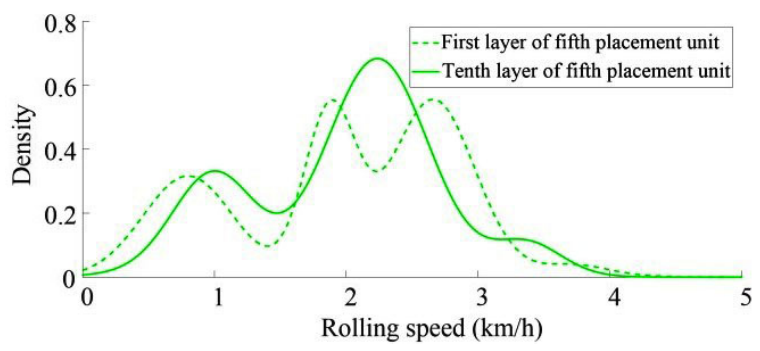

(c)

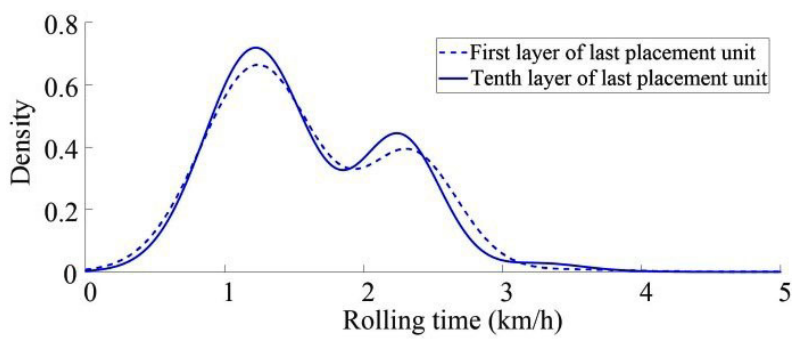

Figure 16. Rolling speed density analysis

going on. This is partly because the roller operators are not familiar with the roller and placement unit environment at the beginning. With constantly alarmed by real-time monitoring system, operators try to a relatively small and steady rolling speed to guarantee compaction quality. In addition, the difference between rolling speed density at the end of first layer and tenth layer gradually decreases, which means the density becomes steady. This also shows that rolling operation level is improving.

\subsection{Dynamic simulation results}

Here, construction area I is taken as an example to illustrate the dynamic simulation process. Construction area I contains 14 placement units, which lasts from 6:32 July 3rd, 2015 to 20:14 December 29th, 2015. Excluding construction interval time between placement units and construction suspension time due to rain or equipment failure, the actual placement unit construction time is 1004.56 hours.

The construction process of area I is simulated by applying parameters that updated after completion of the first, seventh, and last placement unit respectively. Simulation under each set of parameters is conducted 50 times, the simulated durations and their differences to actual 


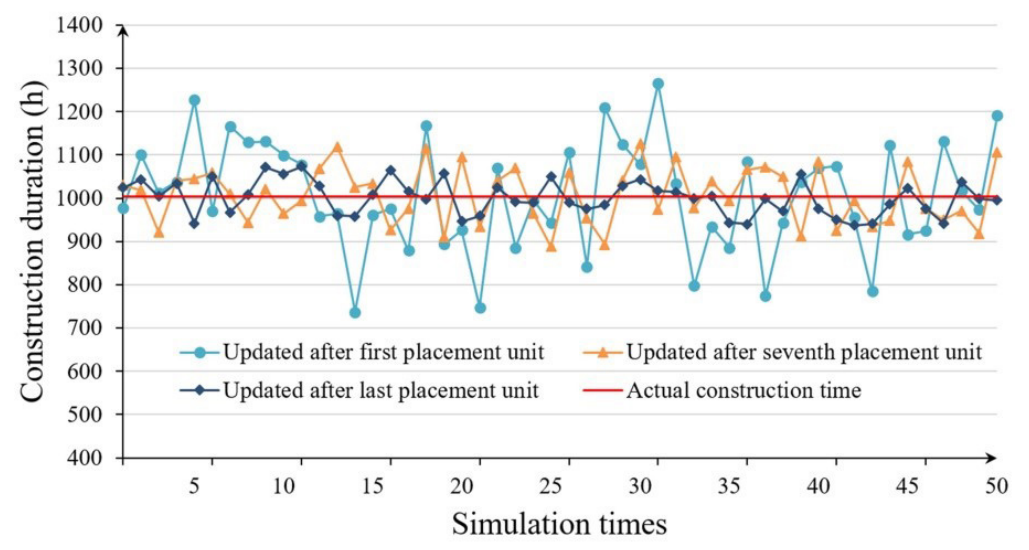

Figure 17. Simulation under different parameters

duration are shown in Figure 17. It's obvious that simulated durations with parameters updated after the last placement unit has the smallest deviations to the actual construction time, compared with ones that using parameters updated after first and seventh placement unit. To measure the deviation level, deviation rate is defined as

$$
D=\sum_{i=1}^{n} \frac{\left|T_{i}-T_{a}\right|}{n T_{a}},
$$

where $n$ is the simulation times, here $n=50 ; T_{i}$ is the simulated duration; $T_{a}$ is the actual duration.

The deviation rates of simulation updated after completion of the first, seventh, and last placement unit are $8.7 \%$, $4.8 \%$, and $2.8 \%$ respectively. The durations using parameters updated after completion of the first placement unit are with higher randomness and their differences to actual duration are relatively large. As the parameters updated with more real-time construction data, the differences decrease and this decrease becomes trivial between update of seventh and last placement unit. The above analysis demonstrates that the accuracy of construction area simulation increases as more real-time data taken into density estimation and parameter update. What's more, it's also proved that the proposed refined simulation model can describe the actual construction process accurately with suitable choice of simulation parameters.

Dynamic simulations of placement unit 2\# to placement unit $14 \#$ in construction area I are also analysed. Each placement unit is simulated 50 times under two parameter update modes. The mode one using parameters updated based on all previous placement units' real-time data. Considering parameter density's dynamic, mode two using parameters updated by only the last placement unit's real-time data is also conducted.

Taking the simulation of placement unit 2\# as an example, there are only placement unit 1\#'s real-time data available for parameters update where two modes are identical. The horizontal layer area of placement unit 2\# is $3729.36 \mathrm{~m}^{2}$ and the maximum constructable area is $4200 \mathrm{~m}^{2}$, so horizontal layer construction is first chosen. The placement unit has a height of three meters, which is divided into ten horizontal layers of $0.3 \mathrm{~m}$ thickness. The width of the placement unit is $36 \mathrm{~m}$, so each layer is further divided into three construction sections and flow process construction is adopted. Each layer's inner-layer activities including paving, rolling, and quality inspection are simulated. The rolling times ratio of every layer's first time rolling is larger than $95 \%$, so there is no additional rolling. The total inner-layer construction time according to Eqns (13) and (14) is 56.97 hours. Inter-layer activities includes mortar paving before the first layer costing 1.24 hours, cool pipes layout after the fifth layer costing 3.42 hours, and bedding mortar paving before the sixth and ninth layer costing 6.52 hours. The whole placement unit construction time is 68.15 hours.

The simulated durations are described by boxplot in Figure 18. According to the overall distribution characteristics, there are three duration change points, i.e. placement unit $4 \#, 8 \#$, and $11 \#$, that placement unit's duration has considerable difference with the last one. It is also proved by the placement unit's deviation rates to actual duration in Table 3 , where deviation rate also changes a lot at placement unit $4 \#, 8 \#$, and $11 \#$. These change points reflect the actual construction conditions changes, based on which the placement units are partitioned into three phases in Figure 18. Phase one including placement unit 2\#, 3\# is a dynamic construction phase. The placement unit durations in phase one vary and the deviation rates are relatively large, which means it's difficult to accurately forecast duration based on precedent construction data. Phase two including placement unit 4\#, 5\#,6\#, 7\#, phase three including placement unit $8 \#, 9 \#$, and 10\#, and phase four including placement unit 11\#, 12\#, 13\#, and 13\# are steady construction phases where the duration distributions are similar. Placement units in the steady phase except the first placement unit have relatively small deviation rates, which means the construction is steady and the simulation parameters updated by precedent real-time data is representative to actual construction process. The first placement unit in the steady phase, i.e. $4 \#, 8 \#$, and $11 \#$, has the larger deviation rate, which is due to the variation of construction conditions to the previous phase. 


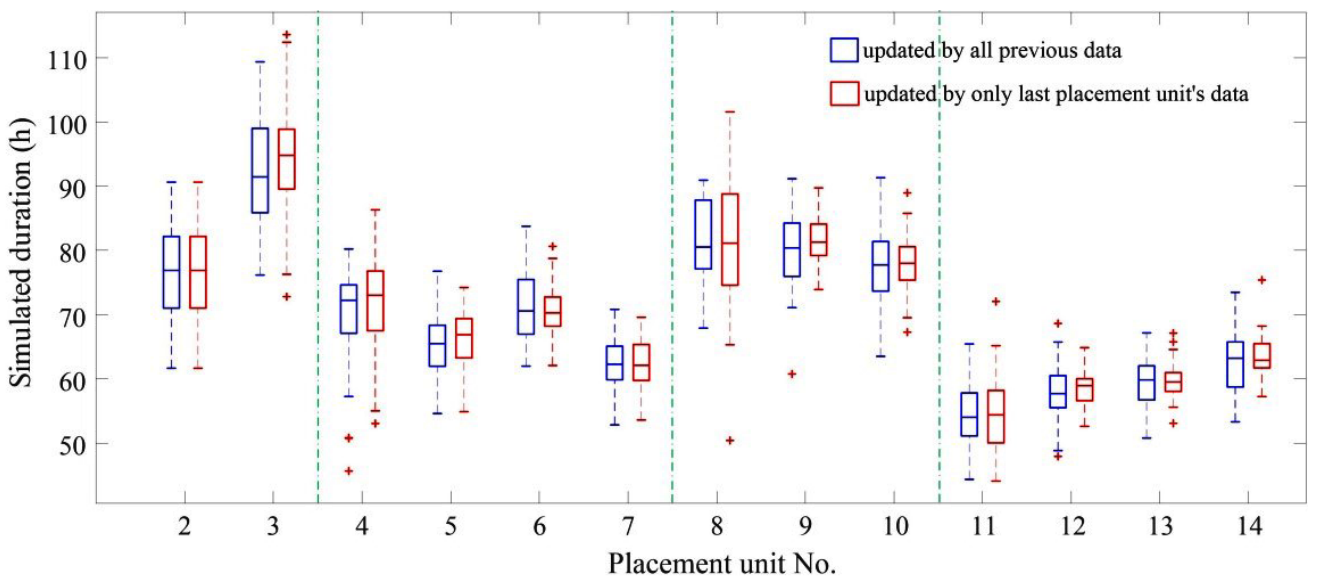

Figure 18. Simulated durations' distribution

Details of simulation results are listed in Table 3, where actual duration, simulation duration mean, and simulation duration's 95\% confidence interval are also given. Apart from placement unit 3\# under simulation mode two, all simulation duration's $95 \%$ confidence intervals contain the corresponding actual duration, which proves that the proposed method can predict the duration accurately. As for Unit 3\#, it's at the dynamic construction phase that its condition is different from Unit 2\#, so using the parameters that updated only based on construction data of Unit 2\# causes the misleading schedule prediction.

In the dynamic phase where construction conditions vary, accurate dynamic simulation is difficult. But the mode one' deviation rates are smaller than mode two's. This is because mode one takes more data into considera- tion in simulation parameter update, which is more adaptable to actual process's dynamic. In the steady phase, mode two's deviation rates are smaller than mode one's. This is because the adjacent placement unit has continuous and consistent construction conditions, considering the all previous placement unit's data including of different phase will bring negative effect to simulation parameter update. From the above analysis, the dynamic simulation based on the last placement unit' construction data can describe the actual process and forecast schedule well in steady construction phase, while the one based on all previous placement units' construction data is more suitable for dynamic construction phase. Both modes of dynamic simulation are recommended in on-site schedule analysis to cope with different situations.

Table 3. Placement unit simulation results

\begin{tabular}{|c|c|c|c|c|c|c|c|c|c|}
\hline \multirow{3}{*}{$\begin{array}{l}\text { Unit } \\
\text { No. }\end{array}$} & \multirow{3}{*}{$\begin{array}{l}\text { Actual } \\
\text { duration } \\
(\mathrm{h})\end{array}$} & \multicolumn{4}{|c|}{ Simulation mode one } & \multicolumn{4}{|c|}{ Simulation mode two } \\
\hline & & \multirow{2}{*}{$\begin{array}{l}\text { Mean } \\
(\mathrm{h})\end{array}$} & \multicolumn{2}{|c|}{ 95\% confidence interval } & \multirow{2}{*}{$\begin{array}{c}\text { Deviation } \\
\text { rate }(\%)\end{array}$} & \multirow{2}{*}{$\begin{array}{c}\text { Mean } \\
\text { (h) }\end{array}$} & \multicolumn{2}{|c|}{ 95\% confidence interval } & \multirow{2}{*}{$\begin{array}{c}\text { Deviation } \\
\text { rate }(\%)\end{array}$} \\
\hline & & & $\begin{array}{c}\text { Lower } \\
\text { limit }(\mathrm{h})\end{array}$ & Upper limit (h) & & & $\begin{array}{c}\text { Lower } \\
\text { limit }(\mathrm{h})\end{array}$ & $\begin{array}{l}\text { Upper limit } \\
\text { (h) }\end{array}$ & \\
\hline $1 \#$ & 92.83 & * & * & * & * & * & * & * & * \\
\hline 2\# & 74.6 & 76.27 & 74.16 & 78.38 & 8.62 & 76.27 & 74.16 & 78.38 & 8.62 \\
\hline $3 \#$ & 90.36 & 92.11 & 89.60 & 94.62 & 8.1 & 94.64 & 92.00 & 97.29 & 9.07 \\
\hline $4 \#$ & 72.04 & 70.25 & 68.10 & 72.40 & 7.44 & 72.09 & 69.98 & 74.19 & 7.7 \\
\hline $5 \#$ & 66.51 & 65.60 & 64.14 & 67.05 & 6.24 & 66.33 & 65.06 & 67.60 & 5.4 \\
\hline $6 \#$ & 70.67 & 71.08 & 69.63 & 72.53 & 5.93 & 70.42 & 69.35 & 71.49 & 4.09 \\
\hline 7\# & 62.56 & 62.34 & 61.14 & 63.53 & 5.36 & 62.26 & 61.21 & 63.31 & 4.8 \\
\hline $8 \#$ & 81.38 & 81.51 & 79.69 & 83.34 & 6.71 & 81.58 & 78.62 & 84.55 & 9.77 \\
\hline 9\# & 80.7 & 80.28 & 78.60 & 81.96 & 5.8 & 81.44 & 80.42 & 82.46 & 3.59 \\
\hline $10 \#$ & 77.21 & 77.27 & 75.60 & 78.94 & 6.03 & 77.89 & 76.70 & 79.07 & 4.35 \\
\hline $11 \#$ & 54.97 & 54.42 & 53.12 & 55.73 & 6.73 & 54.97 & 53.35 & 56.59 & 8.46 \\
\hline 12\# & 58.05 & 57.91 & 56.68 & 59.14 & 5.82 & 58.38 & 57.60 & 59.15 & 3.78 \\
\hline 13\# & 59.37 & 59.23 & 58.06 & 60.29 & 5.41 & 59.75 & 58.99 & 60.50 & 3.48 \\
\hline 14\# & 63.29 & 62.66 & 61.50 & 63.82 & 5.26 & 63.40 & 62.52 & 64.29 & 3.65 \\
\hline
\end{tabular}


Table 4. Simulation of different construction areas

\begin{tabular}{|c|c|c|c|c|c|}
\hline & & & \multicolumn{2}{|c|}{$\begin{array}{c}\text { 95\% Confidence } \\
\text { interval }\end{array}$} & \\
\cline { 4 - 5 } $\begin{array}{c}\text { Area } \\
\text { No. }\end{array}$ & $\begin{array}{c}\text { Actual } \\
\text { duration } \\
\text { (h) }\end{array}$ & \multirow{2}{*}{$\begin{array}{c}\text { Mean } \\
\text { (h) }\end{array}$} & $\begin{array}{c}\text { Lower } \\
\text { limit } \\
\text { (h) }\end{array}$ & $\begin{array}{c}\text { Upper } \\
\text { limit (h) }\end{array}$ & rate (\%) \\
\hline I & 66.51 & 65.60 & 64.14 & 67.05 & 6.24 \\
\hline II & 28.04 & 29.17 & 27.96 & 30.16 & 6.39 \\
\hline III & 35.42 & 34.43 & 33.52 & 35.94 & 5.78 \\
\hline IV & 83.94 & 82.36 & 79.87 & 84.25 & 4.43 \\
\hline V & 41.91 & 42.31 & 41.23 & 42.89 & 3.74 \\
\hline VI & 50.31 & 49.67 & 48.17 & 51.11 & 4.56 \\
\hline VII & 26.83 & 27.85 & 26.39 & 28.35 & 6.98 \\
\hline VIII & 96.74 & 96.31 & 95.02 & 97.60 & 4.39 \\
\hline IX & 90.37 & 88.64 & 86.24 & 90.97 & 3.91 \\
\hline X & 101.35 & 103.76 & 99.70 & 106.42 & 4.03 \\
\hline
\end{tabular}

As Figure 9 showed, there are 10 construction areas of HD RCC dam. To test the simulation method in different construction periods, the fifth placement unit of each area's is simulated. Paving speed, rolling speed, and rolling deviation angle are updated in each area alone, while unit time of quality inspection, mortar paving, cooling pipes layout and bedding mortar paving are updated since the start of the first placement unit of whole dam. The simulated duration and the actual placement unit construction time are showed in Table 4. Each confidence interval $95 \%$ of simulation duration contains the actual duration and the deviation rate is between $3-7 \%$, which demostrates that the simulation method is also applicable to other construction areas.

\section{Conclusions}

In this paper, a construction phase oriented dynamic simulation method for the RCC dam placement process is proposed. The main achievements of this paper are listed as follows:

1. Considering the features of the actual placement process, a detailed simulation model is built that encompasses different organizational forms such as horizontal- or slopping-layer construction and sequence or flow-process construction among multiple activities. In addition to inner-layer activities including paving, rolling, and quality inspection, inter-layer activities including mortar paving, cooling pipes layout, and bedding mortar paving are also considered.

2. To make the simulation model adaptable to the dynamic changes of actual processes, a construction activity modeling method based on real-time monitoring data is proposed. Rolling, as the key activity of the placement process, is given a discrete time model that analyzes the standard rolling time ratio and calculates the additional remedial rolling time.
3. To describe the multipeak and time-variated construction parameter distribution, DPM models are applied to accurately update the simulation parameter density in the infinite-dimensional space. Considering that typical MCMC-based inferences for DPM models are time-consuming, an improved fast inference algorithm coupling PE with SUGS is proposed. The proposed algorithm is compared with SUGS and kernel density estimations and shows good performance.

Based on the above three points and real-time monitoring techniques, a construction phase-oriented dynamic simulation framework is built.

The proposed method is applied to an actual RCC dam project in southwest China, and the results show that the simulation can sensitively capture the features of actual placement processes and accurately forecast on-site near-future schedules. The proposed method not only achieves dynamic schedule prediction and analysis in the construction phase of RCC dam placement processes but also provides a feasible simulation framework that can be conducted for other dynamic multiple-activity processes in projects such as building rock-filled dams and highway construction.

Based on the aforementioned conclusions, further research can be carried out to expand real-time monitoring to collect more kinds of data. Currently, the scope of RCC dam storehouse surface construction real-time monitoring is still limited, mainly focusing on rolling process control. More construction data can be captured by equipping various sensors on construction machines. Then, with more data, construction activities such as paving can be modeled in detail, which will further improve simulation precision. Additionally, developing a human-computer interaction program is critical for the realization of on-site practical applications.

\section{Acknowledgements}

This work was supported by the Innovative Research Groups of the National Natural Science Foundation of China under Grant [number 51621092]; and the National Natural Science Foundation of China under Grant [number 51439005].

\section{Author contributions}

Wei $\mathrm{Hu}$ and Denghua Zhong conceived the study and were responsible for the design and development of the data analysis. Wei $\mathrm{Hu}$ and Zheng Li were responsible for data collection and analysis. Wei $\mathrm{Hu}$ and Binping $\mathrm{Wu}$ were responsible for data interpretation. Wei Hu wrote the first draft of the article.

\section{Disclosure statement}

All authors have no conflict of interest. 


\section{References}

Abdallah, M., \& Marzouk, M. (2013). Planning of tunneling projects using computer simulation and fuzzy decision making. Journal of Civil Engineering and Management, 19(4), 591-607. https://doi.org/10.3846/13923730.2013.793608

AbouRizk, S. M., \& Hague, S. (2009). An overview of the COSYE environment for construction simulation. In Proceedings of the 2009 Winter Simulation Conference (WSC) (pp. 2624-2634). https://doi.org/10.1109/WSC.2009.5429307

AbouRizk, S., \& Mohamed, Y. (2000). Symphony - an integrated environment for construction simulation. In Proceedings of the 2009 Winter Simulation Conference (WSC) (pp. 19071914). https://doi.org/10.1109/WSC.2000.899185

Akhavian, R., \& Behzadan, A. H. (2012). An integrated data collection and analysis framework for remote monitoring and planning of construction operations. Advanced Engineering Informatics, 26(4), 749-761.

https://doi.org/10.1016/j.aei.2012.04.004

Akhavian, R., \& Behzadan, A. H. (2013). Knowledge-based simulation modeling of construction fleet operations using multimodal-process data mining. Journal of Construction Engineering and Management, 139(11), 04013021. https://doi.org/10.1061/(ASCE)CO.1943-7862.0000775

Akhavian, R., \& Behzadan, A. H. (2015). Construction equipment activity recognition for simulation input modeling using mobile sensors and machine learning classifiers. Advanced Engineering Informatics, 29(4), 867-877. https://doi.org/10.1016/j.aei.2015.03.001

Alvanchi, A., Lee, S. H., \& AbouRizk, S. (2011). Modeling framework and architecture of hybrid system dynamics and discrete event simulation for construction. Computer-Aided Civil and Infrastructure Engineering, 26(2), 77-91. https://doi.org/10.1111/j.1467-8667.2010.00650.x

Antoniak, C. E. (1974). Mixtures of dirichlet processes with applications to Bayesian nonparametric problems. Annals of Statistics, 2(6), 1152-1174. https://doi.org/10.1214/aos/1176342871

Bandt, C., \& Pompe, B. (2002). Permutation entropy: a natural complexity measure for time series. Physical Review Letters, 88(17), 174102.

https://doi.org/10.1103/PhysRevLett.88.174102

Chung, T. H., Mohamed, Y., \& Abourizk, S. (2006). Bayesian updating application into simulation in the north edmonton sanitary trunk tunnel project. Journal of Construction Engineering and Management, 132(8), 882-894. https://doi.org/10.1061/(ASCE)0733-9364(2006)132:8(882)

Darema, F. (2004). Dynamic data driven applications systems: A new paradigm for application simulations and measurements. In International Conference on Computational Science (pp. 662-669). https://doi.org/10.1007/978-3-540-24688-6_86

Escobar, M. D. (1994). Estimating normal means with a dirichlet process prior. Journal of the American Statistical Association, 89(425), 268-277.

https://doi.org/10.1080/01621459.1994.10476468

Escobar, M. D., \& West, M. (1995). Bayesian density estimation and inference using mixtures. Journal of the American Statistical Association, 90(430), 577-588. https://doi.org/10.1080/01621459.1995.10476550

Guan, T., Zhong, D. H., Ren, B. Y., Song, W. S., \& Chu, Z. Q. (2018). Construction simulation of high arch dams based on fuzzy bayesian updating algorithm. Journal of Zhejiang University - Science A, 19(7), 505-520. https://doi.org/10.1631/jzus.A1700372
Gurgun, A. P., Zhang, Y., \& Touran, A. (2013). Schedule contingency analysis for transit projects using a simulation approach. Journal of Civil Engineering and Management, 19(4), 465-475. https://doi.org/10.3846/13923730.2013.768542

Halpin, D. W. (1977). CYCLONE - A method for modeling job site process. Journal of the Construction Division, 103(3), 489-499.

Halpin, D. W. (1990). Micro-CYCLONE user's manual. Division of Construction Engineering and Management, Purdue University.

Han, S., Ko, Y. H., Hong, T., Koo, C., \& Lee, S. (2016). Framework for the validation of simulation-based productivity analysis: focused on curtain wall construction process. Journal of Civil Engineering and Management, 23(2), 163-172. https://doi.org/10.3846/13923730.2014.992468

Ioannou, P. G. (1989). UM-CYCLONE user's guide. Department of Civil Engineering, University of Michigan.

Ishwaran, H., \& James, L. F. (2001). Gibbs sampling methods for stick-breaking priors. Journal of the American Statistical Association, 96, 161-173. https://doi.org/10.1198/016214501750332758

Jain, S., \& Neal, R. M. (2004). A split-merge Markov chain Monte Carlo procedure for the Dirichlet Process mixture model. Journal of Computational and Graphical Statistics, 13, 158-182. https://doi.org/10.1198/1061860043001

Law, A. M. (2015). Simulation modeling and analysis (5 $5^{\text {th }}$ ed.). New York: McGraw-Hill.

Lennox, K. P., Dahl, D. B., Vannucci, M., Day, R., \& Tsai, J. W. (2010). A Dirichlet process mixture of hidden Markov models for protein structure prediction. The Annals of Applied Statistics, 4(2), 916-942. https://doi.org/10.1214/09-AOAS296

Liu, Y., Zhong, D., Cui, B., Zhong, G., \& Wei, Y. (2015). Study on real-time construction quality monitoring of storehouse surface for RCC dams. Automation in Construction, 49(1), 100-112. https://doi.org/10.1016/j.autcon.2014.10.003

Lo, A. Y. (1984). On a class of Bayesian nonparametric estimates I: density estimates. The Annals of Applied Statistics, 12, 351-357. https://doi.org/10.1214/aos/1176346412

Lu, M., Dai, F., \& Chen, W. (2007). Real-time decision support for planning concrete plant operations enabled by integrating vehicle tracking technology, simulation, and optimization algorithms. Canadian Journal of Civil Engineering, 34(8), 912-922. https://doi.org/10.1139/107-029

Luo, W., Liu, Q., \& Hu, Z. G. (2009). Study on RCC dam construction system coupling based on Petri net. Journal of System Simulation, 21(7), 2053-2056.

Maceachern, S. N. (1994). Estimating normal means with a conjugate style Dirichlet process prior. Communications in Statistics-Simulation and Computation, 23(3), 727-741. https://doi.org/10.1080/03610919408813196

Martinez, J. C., \& Ioannou, P. G. (1994). General purpose simulation with stroboscope. In Proceedings of the $26^{\text {th }}$ Conference on Winter Simulation (pp. 1159-1166). https://doi.org/10.1109/WSC.1994.717503

Müller, P., Quintana, F. A., Jara, A., \& Hanson, T. (2015). Bayesian nonparametric data analysis. Springer. https://doi.org/10.1007/978-3-319-18968-0

National Economic and Trade Commission of the People's Republic of China. (2001). Design guide of construction equipment selection for hydropower and water conservancy project (DL/T 5133-2001). Beijing, China. 
Oloufa, A. A. (1993). Modeling operational activities in objectoriented simulation. Journal of Computing in Civil Engineering, 7(1), 94-106. https://doi.org/10.1061/(ASCE)0887-3801(1993)7:1(94)

Pereira, V., Ferré, G., Giremus, A., \& Grivel, E. (2014). Relevance of Dirichlet process mixtures for modeling interferences in underlay cognitive radio. In Proceedings of the $22^{\text {nd }}$ European Signal Processing Conference (EUSIPCO) (pp. 176-180).

Rabaoui, A., Viandier, N., Duflos, E., Marais, J., \& Vanheeghe, P. (2012). Dirichlet process mixtures for density estimation in dynamic nonlinear modeling: Application to GPS positioning in urban canyons. IEEE Transactions on Signal Processing, 60(4), 1638-1655.

https://doi.org/10.1109/TSP.2011.2180901

Son, J., Rojas, E. M., \& Shin, S. W. (2015). Application of agentbased modeling and simulation to understanding complex management problems in CEM research. Journal of Civil Engineering and Management, 21(8), 998-1013. https://doi.org/10.3846/13923730.2014.893916

Song, L. G., \& Eldin, N. N. (2012). Adaptive real-time tracking and simulation of heavy construction operations for lookahead scheduling. Automation in Construction, 27(6), 32-39. https://doi.org/10.1016/j.autcon.2012.05.007

Tsiligkaridis, T., \& Forsythe, K. (2015). Adaptive low-complexity sequential inference for Dirichlet process mixture models. In NIPS'15 Proceedings of the $28^{\text {th }}$ International Conference on Neural Information Processing Systems (pp. 28-36). MA, USA: MIT Press Cambridge.

Vahdatikhaki, F., \& Hammad, A. (2014). Framework for near real-time simulation of earthmoving projects using location tracking technologies. Automation in Construction, 42(42), 50-67. https://doi.org/10.1016/j.autcon.2014.02.018
Wang, L., \& Dunson, D. B. (2011). Fast Bayesian inference in Dirichlet process mixture models. Journal of Computational and Graphical Statistics, 20(1), 196-216. https://doi.org/10.1198/jcgs.2010.07081

Wang, Q. W., Zhong, D. H., Wu, B. P., Yu, J., \& Chang, H. T. (2018). Construction simulation approach of roller-compacted concrete dam based on real-time monitoring. Journal of Zhejiang University - Science A, 19(5), 367-383. https://doi.org/10.1631/jzus.A1700042

Wang, R. C., Zhong, D. H., \& Zha, J. M. (1995). Simulation study on the construction process of high roller compacted concrete dam. Journal of Hydroelectric Engineering, 1, 25-37.

West, M., \& Escobar, M. D. (1993). Hierarchical priors and mixture models, with application in regression and density estimation. Institute of Statistics and Decision Sciences, Duke University.

Zhang, S., Du, C., Sa, W., Wang, C., \& Wang, G. (2014). Bayesian-based hybrid simulation approach to project completion forecasting for underground construction. Journal of Construction Engineering and Management, 140(1), 04013031. https://doi.org/10.1061/(ASCE)CO.1943-7862.0000764

Zhao, C. J., Dong, H., \& Zhou, Y. H. (2013). Study on simulation and optimization of concrete placement system on construction surface of RCC dam. Water Resources and Hydropower Engineering, 44(1), 79-82.

Zhao, Y., Kang, J., \& Yu, T. (2014). A Bayesian nonparametric mixture model for selecting genes and gene subnetworks. The Annals of Applied Statistics, 8(2), 999-1201.

https://doi.org/10.1214/14-AOAS719 